\title{
La etnobotánica Aonik'enk (Tehuelches de Santa Cruz, Argentina) inédita de Raúl Martínez Crovetto: FITONIMIA, CORRESPONDENCIAS BOTÁNICAS Y ANÁLISIS NOMENCLATURAL \\ The unpublished AoniK'EnK (Tehuelches from Santa Cruz, Argentine) ethnobotany of Raúl Martínez Crovetto: Plant names, BOTANICAL CORRESPONDENCES AND NOMENCLATURAL ANALYSIS
}

\author{
Gustavo F. Scarpa ${ }^{1 *}\left(\mathbb{C}\right.$, Leonardo M. Anconatani ${ }^{2}$, Cintia N. Rosso ${ }^{1}$ y y \\ Néstor C. D. Huircapán ${ }^{3}$ (D)

\section{SUMMARY}

1. División Botánica del Museo Argentino de Ciencias Naturales "Bernardino Rivadavia" (MACNCONICET) Av. Ángel Gallardo 470, C1405DJR, C.A.B.A., Argentina.

2. Museo de Farmacobotánica "Juan A. Domínguez", Univ. Bs. As., Junín 930, C.A.B.A., Argentina.

3. Centro Universitario de Idiomas, Univ. Bs. As., Av. San Martín 4453, C.A.B.A., Argentina.

*gustavo22et@yahoo.com.ar

\section{Citar este artículo}

SCARPA, G. F., L. M. ANCONATANI, C. N. ROSSO \& N. C. D. HUIRCAPÁN. 2020. La etnobotánica Aonik'enk (Tehuelches de Santa Cruz, Argentina) inédita de Raúl Martínez Crovetto: Fitonimia, correspondencias botánicas y análisis nomenclatural. Bol. Soc. Argent. Bot. 55: 137-157.

DOI: https://doi. org/10.31055/1851.2372.v55. n1.25243

Recibido: 3 Octubre 2019 Aceptado: 22 Noviembre 2019 Publicado: 25 Marzo 2020 Editora: Norma Hilgert (D)
Background and aims: At the Instituto de Botánica del Nordeste (IBONE) valuable unpublished data of Raúl Martínez Crovetto (M.C.) -pionner of the ethnobotany research in Argentina- about the Aonik'enk ethnobotany registered during 1967 at the Santa Cruz province, were found. The aims of this contribution are to analyse and interpret the voluminous set of information that M.C. has surveyed about the Aonik'enk plant names, in order to contribute to the cultural heritage of this indigenous people, and to the understanding of its ethnobotany.

M\&M: The methodology used is that corresponding to "historical ethnobotany", which considers data from the past as a source of primary information on which the classical ethnobotanical method is applied.

Results: It is shown that M.C. register 105 Aonik'enk plant names used to name 165 botanical taxa (mostly -88.5 \%- native plants) in a total of 236 correspondences among them. Semantic analysis of the vernacular nomenclature results in 45 primary lexemes; 10 secondary lexemes, and 28 metaphorical or descriptive names, besides of 22 refonologized loans (absolute and from translation) acquired from other languages.

Conclusions: It is worth highlighting the setting in value, the analysis and the ethnotaxonomic implications of the numerous information referred to, as well as its updating and the original data provided by the authors.

\section{KEY WORDS}

Vernacular nomenclature, Patagonia, Historical Ethnobotany.

\section{RESUMEN}

Introducción y objetivos: En el Instituto de Botánica del Nordeste (IBONE) se hallaron valiosos datos inéditos de Raúl Martínez Crovetto (M.C.) -pionero de las investigaciones etnobotánicas en Argentina- sobre la etnobotánica aonik'enk registrados durante 1967 en la provincia de Santa Cruz. Los objetivos de esta contribución son analizar e interpretar el voluminoso conjunto de informaciones que M.C. ha relevado sobre la fitonimia Aonik'enk, a los fines de contribuir al patrimonio cultural de este pueblo y a la comprensión de su etnobotánica.

M\&M: La metodología empleada es la correspondiente a la "etnobotánica histórica", la cual considera a los datos del pasado como una fuente de información primaria sobre los cuales se aplica el método etnobotánico clásico.

Resultados: Se comprueba que M.C. registra un total de 105 fitónimos aonik'enk que se emplean para denominar a 165 taxa botánicos (la mayoría de ellos -88.5 \%- nativos), en un total de 236 correspondencias. El análisis semántico de la nomenclatura vernácula consigna 45 lexemas primarios, 10 lexemas secundarios y 28 nombres metafórico-descriptivos, además de 22 préstamos de traducción y absolutos re-fonologizados tomados de otras lenguas.

Conclusiones: Se destacan la puesta en valor, el análisis y las implicancias etnotaxonómicas de la cuantiosa información referida, así como su actualización y datos originales aportados por los autores.

Palabras clave

Nomenclatura vernácula, Patagonia, Etnobotánica histórica. 


\section{INTRODUCCIÓN}

En un trabajo anterior acerca de la etnobotánica inédita que Raúl Martínez Crovetto (M.C.) nos legara sobre los indígenas moqoit, los autores destacamos que "la cuantiosa información histórica en términos de nombres indígenas recopilados y de taxa botánicos a los que hacen referencia contextualizada en tiempo y espacio, pone de manifiesto en forma indudable la relevancia que las inéditas investigaciones de M.C. tienen todavía para la etnobotánica argentina actual" (Scarpa \& Rosso, 2014). Esta afirmación se halla aquí confirmada con el hallazgo de nuevos datos inéditos pertenecientes a M.C. sobre la etnobotánica aonik'enk en la biblioteca del IBONE (CONICET-UNNE, Corrientes). Dicha información, que fuera recabada por este autor hace más de 50 años (1967) en la provincia de Santa Cruz, incluye listados detallados de la fitonimia de este pueblo originario asociados a distintos niveles de identificaciones botánicas, así como también datos sobre aplicaciones específicas (usos) de los vegetales.

Los indígenas que habitaban en la provincia de Santa Cruz fueron históricamente considerados bajo el genérico "tehuelches" (o "patagones") por la mayoría de los autores y particularizados como "de la tierra firme" (Escalada, 1949) o "meridionales" o "del sur" (Casamiquela, 1965). Este último autor distingue entre estos últimos a los "tehuelches meridionales australes" -que habitaban al norte del Estrecho de Magallanes hasta el río Santa Cruz- de los "tehuelches meridionales boreales" -que vivían entre dicho río y el río Chubut-, cuyo idioma era el "teushen" y hoy se hallarían extintos. Los primeros fueron referidos con el etnónimo específico de "aónik(e)nk(e)n" (Spegazzini, 1884), “aóenikenk" (Harrington, 1946), "aônükün 'k" (Vignati, 1936), o "aónik'énk" (Escalada, 1949; Casamiquela, 1965). Escalada -citado por Nacuzzi (2005)- informa que estos eran "nómades cazadores-recolectores de raíces, tubérculos y algunos pocos frutos. Su industria estaba limitada al trabajo de la piedra, al curtido de cuero y a la rústica confección de utensilios de madera" y habitaban preferentemente la estepa patagónica, aunque su rango de nomadismo incluía también la costa marítima y el bosque subantártico.
Domínguez Díaz (2010) afirma que "el contacto interétnico generó tempranamente un sistemático proceso de exterminio principalmente de los aonik'enk y selk'nam, debido a la explotación aurífera, la llegada de los estancieros y la cría intensiva de ganado lanar" y que "las deportaciones a las misiones salesianas alteraron el normal desarrollo cultural de los indígenas que ahí se fueron a vivir". Según Censabella (1999), se desconoce el número de personas pertenecientes a este grupo étnico, variando su estimación de acuerdo a los investigadores. De su lengua, el aonek'o 'ayen o tehuelche (familia lingüística Chon), quedaban hace más de 20 años unos pocos hablantes "que no la usan habitualmente, sino que sólo la recuerdan” (Fernández Garay, 1998). Según el Registro Nacional de Comunidades Indígenas dependiente de la Secretaría de Cultura de la Nación (2018), en la provincia de Santa Cruz existiría un solo núcleo de "tehuelches" propiamente dichos (aonik'enk) en el centro del Departamento Güer Aike $\left(50^{\circ} 65^{\prime}\right.$ lat. S, $70^{\circ} 75^{\prime}$ long. W) y otros tres categorizados como "mapuche-tehuelches" en las inmediaciones de los municipios de Río Gallegos, Puerto Santa Cruz y 28 de Noviembre, de la misma jurisdicción departamental. Según la Coordinación de Educación Intercultural Bilingüe de la Provincia del Chubut (Diario Jornada, 2004) y Daniel Huircapán, en la localidad de Río Mayo quedarían al menos una docena de semi-hablantes o "recordadores de palabras" de dicho idioma.

Si bien contamos con abundantes referencias históricas sobre este grupo étnico provenientes de exploradores e historiadores (compiladas por Martinič, 1995), de corte etnográfico (Escalada, 1949; Siffredi \& Matarrese, 2004; Casamiquela, 2008; entre otros) y lingüístico (Censabella, 1999; Fernández Garay, 2004), los antecedentes sobre su etnobotánica son realmente escasos. En efecto, solo encontramos para los aonik'enk menciones episódicas y fragmentarias sobre algunos pocos usos de plantas identificadas por botánicos en los trabajos de Vera (1991), Martinič (1995) y Rapoport et al. (2001) -compilados por Domínguez Díaz (2010)- que prácticamente no hacen referencia ni a sus nombres indígenas ni a sus respectivas correspondencias botánicas, motivo principal de este artículo. Los diccionarios de Molina (1967) y Fernández Garay (2004) hacen referencia a unos 40 fitónimos aonik'enk -en 


\section{G. F. Scarpa et al.- La etnobotánica Aonik'enk inédita de Raúl Martínez Crovetto}

total- con sus correspondientes nombres botánicos, aunque se desconoce quiénes y cómo se efectuaron dichas determinaciones o de qué obras fue extraída dicha información. Por todo ello, la identificación y análisis de los datos inéditos de M.C. sobre la relación de este pueblo con su entorno vegetal resulta indudablemente del mayor valor para el patrimonio cultural de este pueblo, ya que sería lo más importante que sobrevive de su etnobotánica al presente.

Los estudios sobre la nomenclatura vernácula asignada a las plantas por un pueblo dado poseen una importancia tanto de índole lexicográfica y etnobotánica como etnotaxonómica (Scarpa \& Rosso, 2014). Desde la perspectiva lingüística, cada uno de sus fitónimos constituye una "entrada" léxica de los diccionarios de lenguas indígenas, conjuntamente con sus correspondientes identificaciones botánicas. La clase de fitónimos empleada aporta un dato de valor sobre el significado general de la planta -liquen u hongo- a la cual se hace referencia, ya que se ha registrado que el nivel de precisión referencial de los mismos guarda estrecha relación con la significación de la planta así nombrada. En concreto, ha sido demostrado que los principales vegetales en su cultura se nombran con lexemas primarios (nombres propios) y las de escaso valor con nombres descriptivos o metafóricos (Scarpa, 2007). Desde el punto de vista etnotaxonómico, en cambio, algunos de los fitónimos constituyen verdaderas categorías clasificatorias vernáculas (como algunos nombres genéricos), o bien incluyen en sí mismos las marcas de clase a las que se hallan incluidos, lo cual resulta sumamente relevante para el análisis de su etnotaxonomía (Scarpa \& Rosso, 2014).

Raúl Martínez Crovetto, quien fuera profesor durante veinticuatro años de la cátedra de Botánica Sistemática y Fitogeografía en la Facultad de Ciencias Agrarias de la UNNE, es considerado el pionero de las investigaciones etnobotánicas propiamente dichas en Argentina (Arenas, 1992). La validez y relevancia de los datos inéditos de este autor, específicamente en cuanto a su precisión botánica -y etnográfica-, ya fueron destacadas en otras obras donde algunos de ellos han sido publicados (Viegas Barros, 1995; Pirondo \& Keller, 2012; Scarpa \& Rosso, 2014). Por otra parte, el hecho de que hayan sido registrados hace ya más de 50 años -como los aquí considerados-, le confiere a estos datos una importancia adicional en vistas a las profundas transformaciones demográficas, socio-culturales y de abandono de su lengua que esta etnia ha sufrido.

El objetivo de esta contribución es poner en valor, actualizar y analizar el conjunto de informaciones de M.C. acerca de la fitonimia de los indígenas aonik'enk que hasta hoy permanece inédito, a los fines de contribuir al patrimonio cultural y a la comprensión de la etnobotánica actual de este grupo humano.

\section{Materiales y Métodos}

Los materiales cuya puesta en valor, actualización y análisis se efectúan en este trabajo provienen de manuscritos inéditos de M.C. hallados por Gustavo Scarpa en la Biblioteca del Instituto de Botánica del Nordeste (IBONE) de la ciudad de Corrientes, durante octubre de 2017. Los mismos consisten en un atado de numerosas fichas de cartulina (Fig. 1B-C) y de cuadernos de campo personales de M.C. (Fig. 1D) hallados en cajas de cartón sin rotular. El conjunto de fichas se haya encabezado por una de ellas rotulada como "Fichas nombres tehuelches - latinos", a la cual le siguen otras inicializadas en su borde superior izquierdo por cada fitónimo aonik'enk, hasta la aparición de otra rotulada como "Nombres latino-tehuelches - total 191", a la cual le siguen otras inicializadas en su borde superior izquierdo por un nombre científico. En las primeras, se indicaban las correspondencias botánicas a las cuales corresponden cada uno de los fitónimos indígenas, incluido -en varios casos- un número seguido de la letra "P-" (que luego se constata que refería al número de herbario de cada uno de los ejemplares) $\mathrm{y}$, en ocasiones, el significado en castellano del nombre aonik'enk de cada planta, liquen u hongo. En el segundo conjunto de fichas, cada una de ellas se haya encabezada por un nombre científico incluido el nombre indígena al que hacía referencia, seguido de una somera descripción de una o más aplicaciones (a veces formas de preparación, partes utilizadas y formas de consumo), un número de herbario del mismo tipo que el anterior $\left(\mathrm{P}-\mathrm{N}^{\circ}\right)$ y un número de entrevista. En casos de identificación botánica nula o parcial (hasta nivel de género), se hace mención a su familia botánica y se incluyen características de la 
Bol. Soc. Argent. Bot. 55 (1) 2020
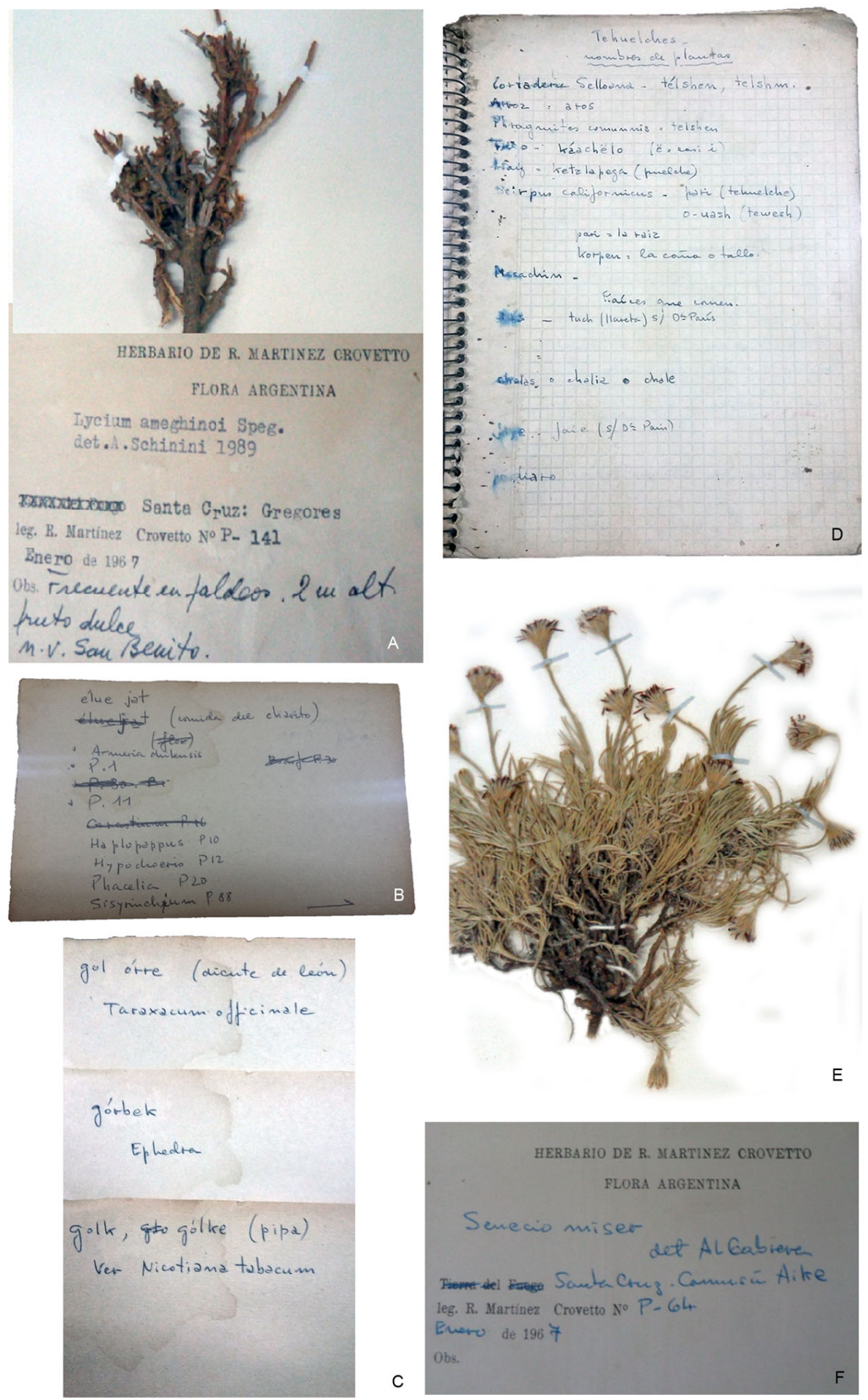

Fig. 1. Documentación original de los datos de M.C. A-E: Ejemplares botánicos coleccionados por M.C. en compañía de aonik'enk depositados en el IBONE. B-C: Fichas de cartulina individuales para cada fitónimo y taxa botánico de M.C. D: Cuaderno de campo personal de M.C. F: Etiqueta de herbario con identificación botánica posterior a M.C. 


\section{G. F. Scarpa et al.- La etnobotánica Aonik'enk inédita de Raúl Martínez Crovetto}

morfología de la planta -liquen u hongo- del tipo: "Comp. chica morada"; "roseta de hoja carnosa"; "Pl. enana Fl. Bl. P. 129” o "Escrof. fl. Lila chica P. 102".

Entre varias libretas de campo originales de M.C. hallamos una rotulada como "Cuaderno de los Tehuelches del sur (Santa Cruz)" o "aonikengs", consistente en un cuaderno espiralado (Fig. 1D). Pudimos comprobar que la información allí registrada resultaba en gran parte similar a los datos consignados en las fichas antes descriptas. En ellos figuran transcriptos los datos recabados a campo para cada una de las entrevistas realizadas, las cuales se hallaban encabezadas por un número, el nombre completo de la persona entrevistada, la localidad y fecha de obtención de los mismos y una cantidad antecedida del signo “\$”, que correspondería al pago que M.C. le habría propinado al colaborador. En total figuran 18 entrevistas efectuadas a 18 colaboradores consultados durante el mes de enero del año 1967 en localidades y parajes de la provincia de Santa Cruz que se localizan en el mapa de la Fig. 2. Luego de ordenar y sistematizar la información extraída de las fichas en una base de datos que responde al programa MS-Access, se efectuó una constatación detallada de cada uno de los datos allí referidos con aquellos obrantes en los cuadernos, lo cual redundó en información que resultó suplementaria a la que fuera hallada en las fichas.

Por otra parte, se efectuó una búsqueda pormenorizada de los ejemplares vegetales citados en las fichas coleccionados en compañía de los entrevistados aonik'enk por M.C., tanto en el Herbario de Plantas Vasculares del IBONE (CTES) (provincia de Corrientes), como a través de la consulta de la base de datos del Instituto de Botánica "Darwinion" (SI) de San Isidro (provincia de Buenos Aires). Esto último se efectuó tanto a los fines de poder citar el material vegetal que documenta las informaciones etnobotánicas que aquí se analizan, como a los fines de obtener identificaciones botánicas más actualizadas a las citadas por M.C., teniendo en cuenta que los materiales habían sido depositados en dichos herbarios hace más de 50 años.

Para analizar toda esta información se empleó el método propio de la "etnobotánica histórica", el cual considera a los datos del pasado como una fuente de información primaria sobre los cuales

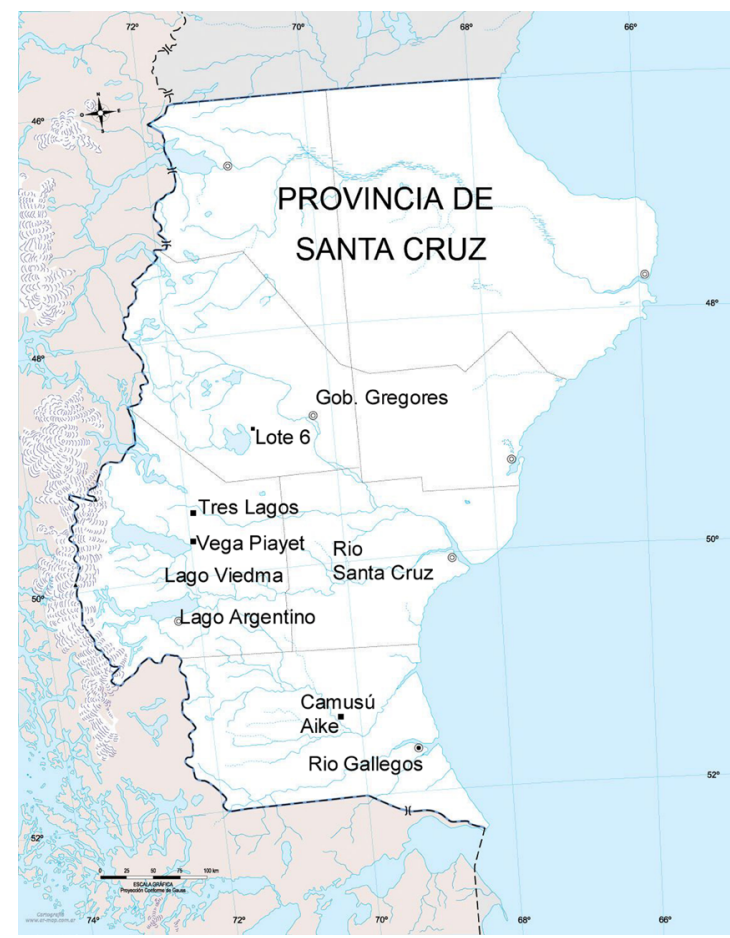

Fig. 2. Mapa de las localidades donde M.C. efectuó las entrevistas etnobotánicas.

se aplica el método clásico etnobotánico (ver Rosso, 2012 y Rosso \& Scarpa, 2012). Mediante este método se procura trascender la simple tarea editorial y de ordenamiento de la información histórica, al contextualizar los datos presentados $\mathrm{y}$ analizados desde las perspectivas espaciotemporal y socio-cultural, al actualizar, confirmar y ampliar las identificaciones botánicas de los taxa referidos y al efectuar un análisis lingüísticonomenclatural somero de la fitonimia referida.

En cuanto a las identificaciones botánicas señaladas por M.C. se procedió a verificar la validez del binomio, su inclusión como "aceptado" para la base de datos Flora Argentina (2018), su distribución en la provincia de Santa Cruz y su actualización en caso de su condición de "sinónimo". Se emplearon para ello las bases de datos Flora del Cono Sur (Zuloaga \& Morrone, 2009), TROPICOS (2009) y The Plant List (2013) para plantas vasculares y GBIF (2019), CABI (2019) y Myco Bank Database (2019) para hongos y líquenes. 
Las categorías semánticas estructurales empleadas aquí para clasificar los fitónimos aonik'enk son las usualmente las empleadas en estudios de esta índole (Friedberg, 1991; Ellen, 2000; Scarpa, 2010), a saber: "lexema primario" (nombres propios) como aquél que contiene una base nominal o "término básico" cuya relación con la planta es puramente arbitraria (i.e. $k^{\prime} o^{\prime} r=$ "pasto"), "lexema secundario" formado por un lexema primario seguido de un calificador que denota cierta parte, propiedad o característica morfológica o utilitaria de la planta a la que este denomina (i.e. $k$ 'o'r poxenk = "pasto oloroso") y "nombre metafórico-descriptivo" consistente en una frase nominal que indica un tipo de asociación con algún atributo de la planta, ya sea de índole morfológico, utilitario, perceptual, fenológico, de hábitat o relación con la fauna, entre otros (i.e. 'ojo $x a: t=$ "comida del ñandú"). A estas se añadió la categoría "préstamo" ya sea cuando el fitónimo referido consiste en el calco semántico del nombre de la planta en español (préstamo de traducción), cuando el préstamo es total o "absoluto" (fitónimo en castellano), o cuando este es parcial o mixto (vocablos aonik'enk y castellano).

A los fines de la segmentación, elicitación y categorización semántica de los fitónimos en idioma aonik'enk cuya correspondencia botánica refiere M.C. se contó con el valioso asesoramiento de Néstor C.D. Huircapán, hablante nativo (originario de las comunidades tehuelches septentrionales australes del Norte de la provincia de Chubut) y docente en el Centro Universitario de Idiomas (C.U.I.) de la Universidad de Buenos Aires del curso de idioma "Tehuelche" o Günün a yajüch (emparentado con el de sus vecinos sureños aonik'enk). También contamos para ello con las traducciones registradas por M.C. en las fichas y con la consulta a los diccionarios en lengua aonik'enk de Molina (1967), Fernández Garay (2004) y de Casamiquela (2008).

Además de citar literalmente la notación fonética de los fitónimos aonik'enk empleada por M.C., la misma fue adecuada a los símbolos empleados por el International Phonetic Alphabet (IPA) siguiendo los esquemas consonánticos y vocálicos referidos en el diccionario de Fernández Garay (2004). Respecto a este tipo de notación se siguen aquí los criterios de esta autora para referir los fonemas diacríticos del aonik'enk distintos al castellano, a saber: " $\mathrm{j”}$ consonante continua, oral, sonora, palatal; "w" consonante continua, oral, sonora, labio-velar; " $\mathrm{t}$ "” consonante oclusiva, oral, glotalizada, sorda, ápico-dental; “č ”" consonante africada, oral, glotalizada, sorda, palatal; “š" consonante fricativa, oral, chicheante, sorda, predorso-prepalatal; " $k$ " consonante oclusiva, oral, glotalizada, sorda, velar; " $x$ " consonante fricativa, oral, sorda, velar; "X" consonante fricativa, oral, sorda, uvular; " " " consonante oclusiva, oral, sorda, glotal y “:” alargamiento vocálico.

El criterio empleado para la cuantificación de los taxa involucrados en el caso que éstos hayan sido identificados hasta el mismo género botánico (i.e. Hordeum spp.) es considerar como diferentes aquellos para los cuales M.C. refirió distintos ejemplares de herbario o que correspondiesen a etnoespecies diferentes (distintos nombres vulgares). Asimismo, para la cuantificación de los fitónimos aonik'enk no se consideran como diferentes las distinas variantes alofónicas identificadas para cada uno de ellos (i.e. teš, tǐ̌s, terš).

\section{Resultados}

\section{El trabajo de campo de Raúl Martínez Crovetto}

El análisis de los cuadernos de campo de M.C. indica que entrevistó a un total de 18 colaboradores de origen aonik'enk en las localidades de Rio Gallegos y Paraje Camusú Aike del Departamento Güer Aike, en Tres Lagos y Paraje Vega Piayet (ex reserva 'Lago Viedma' o 'Lote 119') del Departamento Lago Argentino y en Gobernador Gregores y Paraje Lote 6 (ex reserva 'Lago Cardiel' o 'Lote 6') del Departamento Río Chico, todas ellas en la provincia de Santa Cruz (Fig. 2). La totalidad de las entrevistas fueron realizadas en un solo viaje de campo durante enero del año 1967. En dichos cuadernos M.C. registró los datos etnobotánicos obtenidos de cada uno de los entrevistados, indicando como acápite para cada uno de ellos su nombre, localidad, fecha, número de entrevista y un monto dinerario (el cual rondaba entre los $\$ 100$ y $\$ 300$ de esa época e indicaría el usual pago a los colaboradores durante el trabajo de campo). Dentro de cada uno de ellos, a su vez, la información etnobotánica se halla ordenada por el nombre aonik'enk de la planta, liquen u hongo. En algunas ocasiones figura el nombre botánico científico correspondiente/s a cada 


\section{G. F. Scarpa et al.- La etnobotánica Aonik'enk inédita de Raúl Martínez Crovetto}

fitónimo y un número de colecta seguido de la letra "P." (i. e "P. 62").

\section{Identificaciones botánicas}

\subsection{Identificaciones de Martínez Crovetto:}

Se pudo localizar una parte importante de los ejemplares de herbario coleccionados por M.C. en la provincia de Santa Cruz, tanto en el Herbario del IBONE -bajo la sigla CTES- como en la base de datos del Instituto de Botánica Darwinion (IBODA) -bajo la sigla SI-. En las etiquetas de los ejemplares consultados coinciden, además de los datos del colector y la provincia, los de las localidades señaladas en los cuadernos, las fechas (enero de 1967) y el código particular de colecta antes mencionado ("P. $\mathrm{n}$ "). De esto último se desprende, de manera inequívoca, que estos ejemplares constituyen la documentación del material botánico de los datos de las entrevistas antes mencionadas (Fig. 1A-E).

Entre las determinaciones botánicas de M.C se halló un binomio inexistente: "Perezia lanata", el cual consideramos que se trataría de un error de escritura referente a $P$. laciniata (Phil.) Reiche, endémica de la Patagonia argentinochilena, hoy sinónimo de Perezia pedicularidifolia Less. Asimismo, hemos detectado un error en la identificación de uno de los hongos denominado "Äjchun kuajénwi / jájejenwi” como "Calvatia bovista var. magallanica", ya que según las bases de datos consultadas corresponde a un taxón con distribución circunscripta a América del Norte. Es por ello que dejamos consignado su presencia como Calvatia sp.

\subsection{Identificaciones obtenidas por los autores:}

Para ciertos casos en los que la identificación de M.C. llegaba solo hasta género botánico se recurrió a consultar la Flora Patagónica (Correa, 1984) a fin de efectuar una estimación de la especie en cuestión. Tal es el caso del taxón referido por M.C. como "Salicornia sp." (kampter jaten), la cual se trataría indudablemente de Sarcocornia neei (Lag.) M.A. Alonso \& M.B. Crespo, sinónimo actual de la única especie de dicho género que se encuentra en la Patagonia argentina. Para otros casos de determinaciones hasta el nivel de género realizadas por M.C. se proponen identificaciones "a confrontar" (cf.), sugiriendo el epíteto específico más probable de acuerdo a su distribución en la zona según las bases de datos consultadas (i.e. Alstroemeria cf. patagonica Phil.). Una situación especial pudo hallarse respecto a las distintas especies del género Berberis, ya que M.C. se refiere a ellas como "Berberis spp." o "todos los Berberis" al hacer referencia a su nombre vulgar. Este habría coleccionado varias de las especies de este género que crecen en la provincia de Santa Cruz, aunque sin embargo no se pudieron hallar estos ejemplares ni en los herbarios ni en las bases de datos consultadas. Por ello decidimos indicar las especies de Berberis señaladas para el norte de la Patagonia en las libretas de campo por M.C. correspondientes a otras de sus campañas precedido de "cf.", previa confirmación de su presencia en la provincia de Santa Cruz según la Flora Argentina. Asimismo, al consultar los ejemplares vegetales coleccionados por M.C. en la provincia de Santa Cruz bajo la numeración personal "P-n" en el Herbario de Plantas Vasculares del IBONE (CTES), los autores hemos hallado en sus etiquetas novedosas identificaciones botánicas infragenéricas producto del trabajo efectuado por taxónomos desde que aquél allí los depositara (determinaciones de A.L. Cabrera, G. Bernardello, entre otros) (ver Fig. 1A-F). Como producto de la labor antedicha se obtuvieron 42 nuevas identificaciones hasta el nivel de especie y otras 5 (cinco) más sobre material de herbario sin determinar por M.C. ("sin datos" en Tabla 1). En suma, las nuevas identificaciones obtenidas por los autores suman en conjunto 47 (cuarenta y siete) taxa -sin contar las sinonimias actualizadas-, equivalente al $28.4 \%$ de las determinaciones totales.

En conjunto se identificaron 165 taxones botánicos sobre los que fueron registrados fitónimos aonik'enk por M.C. En la primera columna de la Tabla 1 se enlistan sus nombres científicos actualizados así como los referidos en la fuente por M.C. entre paréntesis, a fin de distinguir claramente los datos provenientes de la información histórica primaria de aquellos derivados de la tarea de los autores. Asimismo, se añaden el o los número/s de ejemplar/es de herbario asociado/s $\mathrm{y}$, en ocasiones, la sigla del herbario donde pudieron hallarse algunos de estos últimos que sirven de documentación a este trabajo.

En la Tabla 2 se cuantifican las identificaciones botánicas registradas -tanto a nivel de género como de especie- para hongos, líquenes y plantas 
Tabla 1. Fitonimia Aonik’enk, correspondencias botánicas y análisis léxico-nomenclatural.

\begin{tabular}{|c|c|c|c|}
\hline $\begin{array}{l}\text { Especie actual (Identificación } \\
\text { de Martínez Crovetto) }\end{array}$ & $\begin{array}{l}\text { Fitónimo Aonik'enk } \\
\text { (notación IPA) }\end{array}$ & Significado y fuentes consultadas & $\begin{array}{l}\text { Tipo de } \\
\text { fitónimo }\end{array}$ \\
\hline \multicolumn{4}{|c|}{ FUNGI } \\
\hline \multicolumn{4}{|l|}{ AGARICACEAE } \\
\hline Agaricus sp. & $\begin{array}{l}\text { Ájchen jaát, Ájchun } \\
\text { jat ('o:kč'en xa:t) }\end{array}$ & $\begin{array}{l}\text { "Comida del espíritu agschem" (MC, dA, } \\
\text { M, D). Dícese de una entidad maligna que } \\
\text { emplean estos hongos como materia prima } \\
\text { para realizar pinturas rupestres y faciales. }\end{array}$ & DE \\
\hline \multicolumn{4}{|l|}{ CYTTARIACEAE } \\
\hline Cyttaria spp. & $\begin{array}{l}\text { Cháun } \\
\text { (č'awn) }\end{array}$ & $\begin{array}{l}\text { (MC, D) Según D provendría de } \\
\text { "châutr" (hongo de los árboles) }\end{array}$ & $1^{\circ}(\mathrm{FV})$ \\
\hline \multicolumn{4}{|l|}{ FISTULINACEAE } \\
\hline Fistulina hepatica (Schaeff.) With. & $\begin{array}{l}\text { Châte } \\
\text { (č'ate) }\end{array}$ & $\begin{array}{l}\text { (MC, D) Según D provendría de } \\
\text { "châutr" (hongo de los árboles) }\end{array}$ & $1^{\circ}(\mathrm{FV})$ \\
\hline \multicolumn{4}{|l|}{ FOMITOPSIDACEAE } \\
\hline $\begin{array}{l}\text { Laetiporus portentosus (Berk.) } \\
\text { Rajchenb. (Polyporus eucalyptorum) }\end{array}$ & $\begin{array}{l}\text { Châte } \\
\text { (č'ate) }\end{array}$ & $\begin{array}{l}\text { (MC, D) Según D provendría de } \\
\text { "châutr" (hongo de los árboles) }\end{array}$ & $1^{\circ}(\mathrm{FV})$ \\
\hline \multicolumn{4}{|l|}{ LYCOPERDACEAE } \\
\hline $\begin{array}{l}\text { Calvatia lilacina (Mont. } \\
\text { \& Berk.) Henn. }\end{array}$ & $\begin{array}{l}\text { Ájchün jájejenwi } \\
\text { ('o:kč’en ‘aXenwe) }\end{array}$ & $\begin{array}{l}\text { "Pintura del espíritu agschem" (MC, } \\
\text { D, FG). Según D aludiría a "jenwe" } \\
\text { traducido como 'lápiz de tierra de color". }\end{array}$ & DE \\
\hline Calvatia sp. & $\begin{array}{l}\text { Ájchün jájejenwi } \\
\text { ('o:kč’en ‘aXenwe) }\end{array}$ & Ídem anterior & $\mathrm{DE}$ \\
\hline \multicolumn{4}{|c|}{ LICHENES } \\
\hline \multicolumn{4}{|l|}{ PARMELIACEAE } \\
\hline $\begin{array}{c}\text { Protousnea dusenii (DuRietz) Krog } \\
\text { (Usnea dusenii) }\end{array}$ & $\begin{array}{l}\text { Kak paj (k'ak pax) } \\
\text { Kak pájelink } \\
\text { (k'ak paxtenk) }\end{array}$ & $\begin{array}{c}\text { "Lana de leña", "leña que tiene lana". } \\
\text { Alude al aspecto filamentoso de } \\
\text { estos líquenes (MC, dA, M, FG) }\end{array}$ & DE \\
\hline $\begin{array}{l}\text { Protousnea magellanica (Mont.) } \\
\text { Krog (Usnea magallanica) }\end{array}$ & $\begin{array}{l}\text { Kárro paj } \\
\text { (k'aro pax) }\end{array}$ & "Lana de árbol” (MC, dA, CM, M, D, FG) & $2^{\circ}$ \\
\hline Usnea campestris R.Sant. & $\begin{array}{l}\text { Yenói koót } \\
\text { (yeno: k’o:t) }\end{array}$ & "Raíz de la mata negra" & $2^{\circ}$ \\
\hline Cf. Usnea sp. (sin datos) & $\begin{array}{l}\text { Âshej ta sháke } \\
\text { ('aščex ša:k'e) }\end{array}$ & $\begin{array}{l}\text { "Barba de mata negra" (MC, dA, M, D, FG). } \\
\text { De "sháke": mata negra (Mulguraea tridens, } \\
\text { Verbena sp.); "ta": su y "ashchej": barba }\end{array}$ & $2^{\circ}$ \\
\hline \multicolumn{4}{|c|}{ SPERMATOPHYTA } \\
\hline \multicolumn{4}{|l|}{ ALSTROEMERIACEAE } \\
\hline $\begin{array}{l}\text { Alstroemeria cfr. patagonica Phil. } \\
\text { (Alstroemeria sp., P. 136) }\end{array}$ & Saj (sax) & $\begin{array}{c}\text { Nombre genérico de plantas con tubérculos } \\
\text { comestibles (FG refiere con "sax" } \\
\text { también a los de Oxalis enneaphylla) }\end{array}$ & $1^{\circ}(\mathrm{FV})$ \\
\hline \multicolumn{4}{|l|}{ AMARYLLIDACEAE } \\
\hline Allium cepa L. & $\begin{array}{l}\text { Kárrol jat } \\
\text { (k’aro' xa:t) }\end{array}$ & “Comida del carancho" (MC, dA, M, D, FG) & DE \\
\hline Allium sativum $\mathrm{L}$. & Ach (ač') & $\begin{array}{l}\text { Derivaría de "achame", nombre genérico } \\
\text { dado a todas las plantas bulbosas }\end{array}$ & $1^{\circ}(\mathrm{FV})$ \\
\hline $\begin{array}{c}\text { Tristagma ameghinoi (Speg.) Speg. } \\
\text { Tristagma patagonicum } \\
\text { (Baker) Traub }\end{array}$ & $\begin{array}{l}\text { Áchame } \\
\text { ('ačamel) }\end{array}$ & $\begin{array}{l}\text { "Cebollita". Según D se trataría } \\
\text { de un nombre genérico dado a } \\
\text { todas las plantas bulbosas }\end{array}$ & $1^{\circ}(\mathrm{FV})$ \\
\hline
\end{tabular}




\section{G. F. Scarpa et al.- La etnobotánica Aonik’enk inédita de Raúl Martínez Crovetto}

Continuación Tabla 1

\begin{tabular}{|c|c|c|c|}
\hline $\begin{array}{l}\text { Especie actual (Identificación } \\
\text { de Martínez Crovetto) }\end{array}$ & $\begin{array}{l}\text { Fitónimo Aonik'enk } \\
\text { (notación IPA) }\end{array}$ & Significado y fuentes consultadas & $\begin{array}{l}\text { Tipo de } \\
\text { fitónimo }\end{array}$ \\
\hline \multicolumn{4}{|l|}{ ANACARDIACEAE } \\
\hline $\begin{array}{l}\text { Schinus marchandii F.A. Barkley } \\
\text { (Schinus sp., P. 144) }\end{array}$ & $\begin{array}{l}\text { Têl (t'el) } \\
\text { molle }\end{array}$ & $\begin{array}{l}\text { "Que pincha" (MC). En alusión } \\
\text { a su condición de espinosa. FG } \\
\text { indica que es nombre propio de } \\
\text { la misma especie botánica }\end{array}$ & $\begin{array}{c}1^{\circ} \\
\operatorname{PRE}(a)\end{array}$ \\
\hline \multicolumn{4}{|l|}{ APIACEAE } \\
\hline $\begin{array}{l}\text { Apium graveolens } \mathrm{L} \text {. } \\
\text { Apium prostratum Labill. ex } \\
\text { Vent. (Apium australe) }\end{array}$ & $\begin{array}{l}\text { Galal } \\
\text { (ga:lal) }\end{array}$ & $\begin{array}{l}\text { Nombre genérico asignado a } \\
\text { plantas con hojas comestibles }\end{array}$ & $1^{\circ}(\mathrm{FV})$ \\
\hline $\begin{array}{l}\text { Azorella prolifera (Cav.) } \\
\text { G.M. Plunkett \& A.N. Nicolas } \\
\text { (Mulinum spinosum, P. 63) }\end{array}$ & Tísmen, tésmem & $(\mathrm{MC}, \mathrm{dA}, \mathrm{M})$ & $1^{\circ}$ \\
\hline Azorella sp. (Mulinum sp.) & Kálche (k'alče) & “espinoso" (MC) & $1^{\circ}(\mathrm{FV})$ \\
\hline Azorella sp. (P. 135) & Tésme & $(\mathrm{MC}, \mathrm{dA}, \mathrm{M})$ & $1^{\circ}$ \\
\hline Azorella spp. & $\begin{array}{c}\text { Teřsh, } \\
\text { Tesh, tish (terš,teš) }\end{array}$ & $(\mathrm{MC}, \mathrm{D}, \mathrm{M}, \mathrm{dA})$ & $1^{\circ}$ \\
\hline Bolax gummifera (Lam.) Spreng. & $\begin{array}{c}\text { Teřsh, } \\
\text { Tesh, tish (terš,teš) }\end{array}$ & $(\mathrm{MC}, \mathrm{D}, \mathrm{M}, \mathrm{dA})$ & $1^{\circ}$ \\
\hline Daucus carota L. & $\begin{array}{l}\text { Kâpenk } \\
\text { (q'a:penk) }\end{array}$ & $\begin{array}{l}\text { "Rojo" (en alusión al color de su } \\
\text { tallo engrosado) (MC, dA, M) }\end{array}$ & DE \\
\hline \multicolumn{4}{|l|}{ AQUIFOLIACEAE } \\
\hline Ilex paraguariensis A. St.-Hil. & $\begin{array}{l}\text { Áashken } \\
\text { ('ašk'om) }\end{array}$ & $\begin{array}{l}\text { Aunque MC traduce como "harina" los } \\
\text { diccionarios dG, dA, CM y M refieren } \\
\text { como "olla", por lo cual estimamos que } \\
\text { aludiría al "mate" como recipiente y } \\
\text { en sentido amplio a la yerba mate. }\end{array}$ & $\mathrm{DE}$ \\
\hline \multicolumn{4}{|l|}{ ASTERACEAE } \\
\hline $\begin{array}{c}\text { Artemisia magellanica Sch. Bip. } \\
\text { (P. 18; P. 142) }\end{array}$ & $\begin{array}{c}\text { Am pojsh } \\
\text { ('am poxšk') }\end{array}$ & "Muy hediondo" (MC, FG). & DE \\
\hline Baccharis sp. & Sheř (šer) & "Mata" (MC, M, dA) & $1^{\circ}(\mathrm{FV})$ \\
\hline $\begin{array}{l}\text { Brachyclados caespitosum } \\
\text { (Phil.) Speg. (P. 105) }\end{array}$ & $\begin{array}{l}\text { Tesh, tish } \\
\text { (teš) }\end{array}$ & $(\mathrm{MC}, \mathrm{D}, \mathrm{M}, \mathrm{dA})$ & $1^{\circ}$ \\
\hline $\begin{array}{l}\text { Chuquiraga aurea Skottsb. } \\
\text { (Chuquiraga sp.) (P. 138) }\end{array}$ & $\begin{array}{l}\text { Kálche } \\
\text { (k'alče) }\end{array}$ & “Espinoso” (MC) & $1^{\circ}(\mathrm{FV})$ \\
\hline $\begin{array}{c}\text { Hypochoeris incana } \\
\text { (Hook. \& Arn.) Macloskie } \\
\text { (Hypochoeris sp., P. 12) (SI) }\end{array}$ & $\begin{array}{l}\text { Élue jat } \\
\text { ('elwe xa:t) } \\
\text { kôřr (k'o'r) }\end{array}$ & $\begin{array}{l}\text { "Comida del pichón de } \\
\text { avestruz" (MC, dA, M, D, FG) } \\
\text { "Pasto" }\end{array}$ & $\begin{array}{c}\mathrm{DE} \\
1^{\circ}(\mathrm{FV})\end{array}$ \\
\hline Lactuca sativa L. & $\begin{array}{c}\text { Galal } \\
\text { (ga:lal) }\end{array}$ & $\begin{array}{l}\text { Nombre genérico asignado a } \\
\text { plantas con hojas comestibles }\end{array}$ & $1^{\circ}$ (gen) \\
\hline $\begin{array}{l}\text { Lepidophyllum cfr. } \\
\text { cupressiforme (Lam.) Cass } \\
\text { (Lepidophyllum sp., P. 3) }\end{array}$ & $\begin{array}{l}\text { Sheř } \\
\text { (šer) }\end{array}$ & "Mata" (MC, M, dA) & $1^{\circ}(\mathrm{FV})$ \\
\hline Matricaria discoidea DC. & $\begin{array}{l}\text { Guéut yáuch } \\
\text { (gewt jawč') } \\
\text { Kôř pójenk } \\
\text { (k'o'r poxenk) }\end{array}$ & $\begin{array}{l}\text { "Tabaco del campo" (MC, dA, CM, M, D) } \\
\text { "Pasto oloroso" (MC, dA, M, D, FG) }\end{array}$ & $2^{\circ}$ \\
\hline $\begin{array}{c}\text { Nassauvia aculeata (Less.) Poepp. } \\
\text { \& Endl. var. azorelloides (Speg.) } \\
\text { Cabrera (Nassauvia abbreviata) }\end{array}$ & $\begin{array}{l}\text { Kôŕr } \\
\text { (k'o'r) }\end{array}$ & "Pasto" & $1^{\circ}(\mathrm{FV})$ \\
\hline $\begin{array}{l}\text { Nassauvia axillaris (Lag. } \\
\text { ex Lindl.) D. Don }\end{array}$ & $\begin{array}{c}\text { Áno têr } \\
\text { (a:no t'er) } \\
\text { Cola de piche }\end{array}$ & $\begin{array}{l}\text { "Cola de piche o Mulita" (MC, D, } \\
\text { dA, M, FG). Sería una traducción al } \\
\text { aonik'enk del nombre vulgar criollo } \\
(M C, D, d A, M)\end{array}$ & $\begin{array}{l}\text { PRE (t) } \\
\operatorname{PRE}(a)\end{array}$ \\
\hline
\end{tabular}


Continuación Tabla 1

\begin{tabular}{|c|c|c|c|}
\hline $\begin{array}{l}\text { Especie actual (Identificación } \\
\text { de Martínez Crovetto) }\end{array}$ & $\begin{array}{l}\text { Fitónimo Aonik'enk } \\
\text { (notación IPA) }\end{array}$ & Significado y fuentes consultadas & $\begin{array}{l}\text { Tipo de } \\
\text { fitónimo }\end{array}$ \\
\hline $\begin{array}{c}\text { Nassauvia fueghiana (Speg.) } \\
\text { Cabrera (P. 134) }\end{array}$ & $\begin{array}{l}\text { Kálche } \\
\text { (k'alče) }\end{array}$ & "Espinoso" (MC) & $1^{\circ}(\mathrm{FV})$ \\
\hline $\begin{array}{l}\text { Perezia pedicularidifolia } \\
\text { Less. (Perezia lanata - } \\
\text { inexistente-, P. 137) (SI) }\end{array}$ & $\begin{array}{l}\text { Tesh, tish } \\
\text { (teš) }\end{array}$ & $(\mathrm{MC}, \mathrm{D}, \mathrm{M}, \mathrm{dA})$ & $1^{\circ}$ \\
\hline $\begin{array}{c}\text { Perezia recurvata (Vahl) } \\
\text { Less. (Hook. \& Arn.) Cabrera } \\
\text { (Mustisia sp. , P. 45) (SI) } \\
\text { (Haplopappus sp., P. 10) (SI) }\end{array}$ & $\begin{array}{c}\text { Wésen } \\
\text { (kwesenk) } \\
\text { Teřsh (terš) } \\
\text { Élue jat ('elwe xa:t) }\end{array}$ & $\begin{array}{c}\text { "Que pincha" (MC, dA), o } \\
\text { "afilado" (FG) "que corta" } \\
\text { (MC, D, M, dA) } \\
\text { "Comida del pichón de avestruz" } \\
\text { (MC, dA, M, D, FG). }\end{array}$ & $\begin{array}{l}\mathrm{DE} \\
1{ }^{\circ} \\
\mathrm{DE}\end{array}$ \\
\hline Perezia sp. (P. 68) & $\begin{array}{c}\text { Élwil jat } \\
\text { ('elwel xa:t) }\end{array}$ & “comida del pájaro élwil” (MC, M, FG) & DE \\
\hline $\begin{array}{c}\text { Senecio miser Hook f. } \\
\text { (Senecio sp., P. 47, P. 64) (SI) }\end{array}$ & $\begin{array}{l}\text { KKôř (k'o'r) } \\
\text { Sheř (šer) }\end{array}$ & $\begin{array}{c}\text { "Pasto" } \\
\text { "mata" (MC, M, dA) }\end{array}$ & $1^{\circ}(\mathrm{FV})$ \\
\hline $\begin{array}{c}\text { Senecio neaei DC. } \\
\text { (Senecio sp., P. 79, P. 81) (SI) }\end{array}$ & $\begin{array}{c}\text { Kó'orr (k'o:'r) } \\
\text { Óiwu jat ('ojo xa:t) }\end{array}$ & $\begin{array}{c}\text { "Pasto" (MC, dA, M, FG) } \\
\text { "Comida del ñandú" (Rhea } \\
\text { pennata) (MC, dA, M, D, FG) }\end{array}$ & $\begin{array}{l}1^{\circ}(\mathrm{FV}) \\
\mathrm{DE}\end{array}$ \\
\hline $\begin{array}{l}\text { Senecio patagonicus Hook. } \\
\text { \& Arn. (Senecio sp., P. } 14 \text {, } \\
\text { P. 114, P. 150) (CTES) }\end{array}$ & Kó'orr (k'o:'r) & "Pasto" (MC, dA, M, FG) & $1^{\circ}(\mathrm{FV})$ \\
\hline $\begin{array}{l}\text { Senecio trifurcatus (G. Forst.) } \\
\text { Less. (Senecio sp., P. 4) (CTES) }\end{array}$ & Sheř (šer) & "mata" (MC, M, dA) & $1^{\circ}(\mathrm{FV})$ \\
\hline $\begin{array}{l}\text { Senecio sericeonitens Speg. } \\
\text { (Senecio sp., P. 78) (CTES) }\end{array}$ & $\begin{array}{l}\text { Óiwu jat } \\
\text { ('ojo xa:t) }\end{array}$ & $\begin{array}{l}\text { "Comida del ñandú" (Rhea } \\
\text { pennata) (MC, dA, M, D, FG) }\end{array}$ & $\mathrm{DE}$ \\
\hline Taraxacum gilliesii Hook. \& Arn. & $\begin{array}{l}\text { Gorrn } \\
\text { (go:l 'o:r) }\end{array}$ & $\begin{array}{c}\text { Sería variante alofónica de "go:l" (león) } \\
\text { y “o:r" (diente) (MC, dA, D, FG }\end{array}$ & PRE (t) \\
\hline $\begin{array}{l}\text { Taraxacum officinale F.H. Wigg. } \\
\quad \text { (Taraxacum sp., P. 82) }\end{array}$ & $\begin{array}{l}\text { Márgo } \\
\text { Gol órre (go:l ‘o:r) } \\
\text { Koôř (k'o'r) }\end{array}$ & $\begin{array}{c}\text { Refonologízación de "amargo", } \\
\text { por el sabor de sus hojas que } \\
\text { los aonik'enk consumen crudas } \\
\text { Sería variante alofónica de "go:l" } \\
\text { (león) y "'o:r" (diente) (MC, dA, D, FG) } \\
\text { "Pasto" }\end{array}$ & $\begin{array}{l}\text { PRE } \\
\text { PRE (t) } \\
1^{\circ}(\mathrm{FV})\end{array}$ \\
\hline Taraxacum sp. (P. 90) & Ot ('ot) & $\begin{array}{l}\text { Según MC se trataría del nombre } \\
\text { propio de un ave paseriforme }\end{array}$ & $1^{\circ}$ \\
\hline \multicolumn{4}{|l|}{ BERBERIDACEAE } \\
\hline $\begin{array}{l}\text { cf. Berberis darwinii Hook. } \\
\text { cf. Berberis empetrifolia Lam. } \\
\text { cf. Berberis microphylla G. } \\
\text { Forst. (Berberis spp.) }\end{array}$ & Chorch (čorč) & $(\mathrm{MC}, \mathrm{M}, \mathrm{D}, \mathrm{FG})$ & $1^{\circ}$ \\
\hline \multicolumn{4}{|l|}{ BORAGINACEAE } \\
\hline $\begin{array}{l}\text { Heliotropium sp. } \\
\text { (Meliotropium sp., P. 122) }\end{array}$ & $\begin{array}{l}\text { Óiwu jat } \\
\text { ('ojo xa:t) }\end{array}$ & $\begin{array}{l}\text { "Comida del ñandú" (Rhea } \\
\text { pennata) (MC, dA, M, D, FG) }\end{array}$ & DE \\
\hline Phacelia sp. (P. 20) (CTES) & $\begin{array}{l}\text { Élue jat } \\
\text { ('elwe xa:t) }\end{array}$ & $\begin{array}{l}\text { "Comida del charito" (pichón de } \\
\text { avestruz) (MC, dA, M, D, FG) }\end{array}$ & DE \\
\hline \multicolumn{4}{|l|}{ BRASSICACEAE } \\
\hline Brassica oleracea L. & $\begin{array}{l}\text { Galal } \\
\text { (ga:lal) }\end{array}$ & $\begin{array}{l}\text { Nombre genérico asignado a } \\
\text { plantas con hojas comestibles }\end{array}$ & $1^{\circ}(\mathrm{FV})$ \\
\hline Capsella bursa-pastoris (L.) Medik. & $\begin{array}{l}\text { Gallina jat } \\
\text { (gašina xa:t) }\end{array}$ & "Comida de gallina" (MC) & $\operatorname{PRE}(p)$ \\
\hline $\begin{array}{l}\text { Descurainia antarctica (E. } \\
\text { Fourn.) O.E. Schulz }\end{array}$ & $\begin{array}{c}\text { Kálken } \\
\text { (kalken) } \\
\text { Sheř verdetenk }\end{array}$ & $\begin{array}{c}\text { Sería variante alofónica de } \\
\text { "káltenk" que significa "verde" (FG) } \\
\text { "Mata verde" (MC, dA, M) }\end{array}$ & $\begin{array}{l}\mathrm{DE} \\
2^{\circ}\end{array}$ \\
\hline $\begin{array}{c}\text { Lepidium didymum L. } \\
\text { (Coronopus didymus, P. 6) }\end{array}$ & $\begin{array}{l}\text { Am pojsh } \\
\text { ('am poxšk') }\end{array}$ & "Muy hediondo" (MC, FG) & $\mathrm{DE}$ \\
\hline
\end{tabular}




\section{G. F. Scarpa et al.- La etnobotánica Aonik’enk inédita de Raúl Martínez Crovetto}

Continuación Tabla 1

\begin{tabular}{|c|c|c|c|}
\hline $\begin{array}{l}\text { Especie actual (Identificación } \\
\text { de Martínez Crovetto) }\end{array}$ & $\begin{array}{l}\text { Fitónimo Aonik'enk } \\
\text { (notación IPA) }\end{array}$ & Significado y fuentes consultadas & $\begin{array}{l}\text { Tipo de } \\
\text { fitónimo }\end{array}$ \\
\hline $\begin{array}{l}\text { Lepidium pseudodidymum } \\
\text { Thell. Ex Druce } \\
\text { (Lepidium sp., P. 2) (SI) (CTES) }\end{array}$ & $\begin{array}{l}\text { Gallina jat } \\
\text { (gašina xa:t) }\end{array}$ & "Comida de gallina" (MC) & $\operatorname{PRE}(p)$ \\
\hline \multicolumn{4}{|l|}{ CACTACEAE } \\
\hline $\begin{array}{l}\text { Austrocactus patagonicus } \\
\text { (F.A.C. Weber) Hosseus } \\
\text { (Cereus patagonicus) }\end{array}$ & $\begin{array}{l}\text { Mo } \\
(\mathrm{mo})\end{array}$ & $(\mathrm{MC}, \mathrm{M})$ & $1^{\circ}(\mathrm{FV})$ \\
\hline $\begin{array}{l}\text { Maihueniopsis darwinii } \\
\text { (Hensl.) F. Ritter var. darwinii } \\
\text { (Opuntia darwinii) }\end{array}$ & $\begin{array}{l}\text { Mo } \\
\text { (mo') }\end{array}$ & $(\mathrm{MC}, \mathrm{M})$ & $1^{\circ}(\mathrm{FV})$ \\
\hline \multicolumn{4}{|l|}{ CALCEOLARIACEAE } \\
\hline Calceolaria sp. (P. 67) (CTES) & $\begin{array}{l}\text { Chóker } \\
\text { (čoker) }\end{array}$ & $\begin{array}{l}\text { "Bota" (MC, dA, M, CM, D, FG) En } \\
\text { alusión a la forma de sus flores }\end{array}$ & DE \\
\hline \multicolumn{4}{|l|}{ CALYCERACEAE } \\
\hline Boopis sp. (P. 38; P. 40) & $\begin{array}{l}\text { Kôřr (k'o'r) } \\
\text { Séperr } \\
\text { (seper) }\end{array}$ & $\begin{array}{l}\text { "Pasto" } \\
\text { "Abrojo", "botón" (dA, M) Aludiría a las } \\
\text { infrutescencias en cabezuelas de esta } \\
\text { planta y a su condición de adherencia. }\end{array}$ & $\begin{array}{c}1^{\circ}(\mathrm{FV}) \\
\mathrm{DE}\end{array}$ \\
\hline Boopis sp. (P. 40) & $\begin{array}{l}\text { K(a)ámpter jât(en) } \\
\text { (k'a'mter xa:ten) }\end{array}$ & "Comida de la lagartija" (MC, M, D, FG) & $\mathrm{DE}$ \\
\hline $\begin{array}{c}\text { Boopis spp. (P. 38a) } \\
\text { Gamocarpha australis (Decne.) } \\
\text { S.S. Denham \& Pozner } \\
\text { (Boopis australis, P. 62, } \\
\text { P. 85) (CTES) }\end{array}$ & $\begin{array}{l}\text { Cháucho jat } \\
\text { (čawčo xa:t) }\end{array}$ & "Comida de la perdiz" (MC) & DE \\
\hline $\begin{array}{l}\text { Gamocarpha australis (Decne.) } \\
\text { S.S. Denham \& Pozner } \\
\text { (Boopis australis, P. 62, P. 85) }\end{array}$ & $\begin{array}{l}\text { K(a)ámpter jât(en) } \\
\text { (k'a'mter xa:ten) }\end{array}$ & “Comida de la lagartija" (MC, M, D, FG) & $\mathrm{DE}$ \\
\hline \multicolumn{4}{|l|}{ CAPRIFOLIACEAE } \\
\hline $\begin{array}{c}\text { Valeriana carnosa Sm } \\
\text { (Valeriana sp., P. 77) (CTES) }\end{array}$ & $\begin{array}{l}\text { K(a)ámpter jât(en) } \\
\text { (k'a'mter xa:ten) }\end{array}$ & "Comida de la lagartija" (MC, M, D, FG) & $\mathrm{DE}$ \\
\hline \multicolumn{4}{|l|}{ CARYOPHYLLACEAE } \\
\hline $\begin{array}{l}\text { Cardionema ramosissima } \\
\text { (Weinm.) A. Nelson \& J.F. Macbr. } \\
(\text { Pentacaena sp., P. 144) }\end{array}$ & $\begin{array}{l}\text { Têl } \\
\text { (t'el) }\end{array}$ & & $1^{\circ}$ \\
\hline $\begin{array}{c}\text { Cerastium cf. arvense } \\
\text { (Cerastium sp., P. 16; P. 98) (CTES) }\end{array}$ & $\begin{array}{l}\text { Óiwu jat } \\
\text { ('ojo xa:t) }\end{array}$ & $\begin{array}{l}\text { "Comida del ñandú" (Rhea } \\
\text { pennata) (MC, dA, M, D, FG) }\end{array}$ & DE \\
\hline $\begin{array}{l}\text { Colobanthus cf. lycopodiodes } \\
\text { Griseb. (Colobanthus sp., P. 53) }\end{array}$ & $\begin{array}{l}\text { Tápel } \\
\text { (tapel) }\end{array}$ & $\begin{array}{l}\text { Sería una variante alofónica de "taper", lo } \\
\text { cual según dA, M es "mata de laguna" y } \\
\text { para FG sería "nudo", "muñeca", "tobillo" }\end{array}$ & $\mathrm{DE}$ \\
\hline $\begin{array}{c}\text { Silene sp. } \\
\text { (Melandryum sp., P. 117) (CTES) } \\
\text { (sin datos, P. 73) }\end{array}$ & $\begin{array}{l}\text { Óiwu jat } \\
\text { ('ojo xa:t) }\end{array}$ & $\begin{array}{l}\text { "Comida del ñandú" (Rhea } \\
\text { pennata) (MC, dA, M, D, FG) }\end{array}$ & $\mathrm{DE}$ \\
\hline Stellaria media (L.) Cirillo & $\begin{array}{l}\text { Gallina jat } \\
\text { (gašina xa:t) }\end{array}$ & "Comida de gallina" (MC) & PRE (p) \\
\hline \multicolumn{4}{|l|}{ CHENOPODIACEAE } \\
\hline Atriplex sp. (P. 13) & lókson, ióksön & & $1^{\circ}$ \\
\hline Beta vulgaris $\mathrm{L}$. & $\begin{array}{l}\text { Galal } \\
\text { (ga:lal) }\end{array}$ & $\begin{array}{l}\text { Nombre genérico asignado a } \\
\text { plantas con hojas comestibles }\end{array}$ & $1^{\circ}(\mathrm{FV})$ \\
\hline $\begin{array}{l}\text { Sarcocornia neei (Lag.) M.A. Alonso } \\
\quad \& \text { M.B. Crespo (Salicornia sp.) }\end{array}$ & $\begin{array}{l}\text { Kaámpter jât(en) } \\
\text { (k'a'mter xa:ten) }\end{array}$ & "Comida de la lagartija" (MC, M, D, FG) & $\mathrm{DE}$ \\
\hline
\end{tabular}


Continuación Tabla 1

\begin{tabular}{|c|c|c|c|}
\hline $\begin{array}{l}\text { Especie actual (Identificación } \\
\text { de Martínez Crovetto) }\end{array}$ & $\begin{array}{l}\text { Fitónimo Aonik'enk } \\
\text { (notación IPA) }\end{array}$ & Significado y fuentes consultadas & $\begin{array}{l}\text { Tipo de } \\
\text { fitónimo }\end{array}$ \\
\hline \multicolumn{4}{|l|}{ CYPERACEAE } \\
\hline $\begin{array}{l}\text { Schoenoplectus californicus } \\
\text { (C.A. Mey.) Soják var. californicus } \\
\text { (Scirpus californicus) }\end{array}$ & $\begin{array}{c}\text { Kórpe } \\
\text { (korpe) } \\
\text { Óompe (o:mpe) } \\
\text { Óush (owš) } \\
\text { Pári (pa'ri) }\end{array}$ & $\begin{array}{c}\text { (MC, } \mathrm{dA}, \mathrm{D}) \text { Según } \mathrm{MC} \text { corresponderia } \\
\text { también al nombre propio del } \\
\text { zorrino macho. } \quad(\mathrm{MC}, \mathrm{dA}, \mathrm{FG})\end{array}$ & $\begin{array}{l}1^{\circ} \\
1^{\circ} \\
1^{\circ} \\
1^{\circ}\end{array}$ \\
\hline \multicolumn{4}{|l|}{ EPHEDRACEAE } \\
\hline Ephedra sp. (P. 22) (CTES) & $\begin{array}{l}\text { Górbek oremberr, } \\
\text { órremer ('oremer) } \\
\text { Tish (teš) }\end{array}$ & Nombre propio de una planta según FG & $\begin{array}{l}1^{\circ} \\
1^{\circ}\end{array}$ \\
\hline \multicolumn{4}{|l|}{ ERICACEAE } \\
\hline Empetrum rubrum Vahl ex Willd. & $\begin{array}{c}\text { Murtilla } \\
\text { Pélko (pelko) }\end{array}$ & & $\begin{array}{c}\operatorname{PRE}(a) \\
1^{\circ}\end{array}$ \\
\hline \multicolumn{4}{|l|}{ EUPHORBIACEAE } \\
\hline $\begin{array}{c}\text { Euphorbia collina Phil } \\
\text { (Euphorbia portulacoides) }\end{array}$ & $\begin{array}{l}\text { lóksön } \\
\text { Pichoga }\end{array}$ & $(\mathrm{MC}, \mathrm{M})$ & $\begin{array}{c}1^{\circ} \\
\operatorname{PRE}(a)\end{array}$ \\
\hline \multicolumn{4}{|l|}{ FABACEAE } \\
\hline Adesmia ameghinoi Speg. (P. 57) & $\begin{array}{l}\text { Kaámpter jât(en) } \\
\text { (k'a'mter xa:ten) }\end{array}$ & "Comida de la lagartija” (MC, M, D, FG) & DE \\
\hline $\begin{array}{l}\text { Adesmia boronioides Hook. F. } \\
\text { (P. 15) (SI) }\end{array}$ & $\begin{array}{l}\text { Sheř (šer) } \\
\text { Shóř chạ (šer č'aj) } \\
\text { Kárto (qarto) } \\
\text { Paramela }\end{array}$ & $\begin{array}{c}\text { "Mata" (MC, M, dA) } \\
\text { Provendría de "šer": mata } \\
\text { y “č'aj”: grande (FG) } \\
\text { (MC, FG) } \\
\text { (MC, FG) }\end{array}$ & $\begin{array}{c}1^{\circ}(\mathrm{FV}) \\
2^{\circ} \\
1^{\circ} \\
\operatorname{PRE}(\mathrm{a})\end{array}$ \\
\hline $\begin{array}{l}\text { Adesmia lotoides Hook. f. (P. 89) } \\
\text { Adesmia villosa Hook. f. (P. 124) }\end{array}$ & $\begin{array}{l}\text { Kaámpter jât(en) } \\
\text { (k'a'mter xa:ten) }\end{array}$ & "Comida de la lagartija” (MC, M, D, FG) & DE \\
\hline $\begin{array}{l}\text { Adesmia volckmannii Phil. } \\
\text { (Adesmia campestris, P. 66) }\end{array}$ & $\begin{array}{l}\text { Mo } \\
\left(\mathrm{mo}^{\prime}\right)\end{array}$ & $(\mathrm{MC}, \mathrm{M})$ & $1^{\circ}(\mathrm{FV})$ \\
\hline $\begin{array}{l}\text { Anarthrophyllum rigidum (Gillies } \\
\text { ex Hook. \& Arn.) Hieron. (P. 111) }\end{array}$ & $\begin{array}{l}\text { Chélkot, chílkot } \\
\text { Mata de guanaco }\end{array}$ & $(\mathrm{MC}, \mathrm{M})$ & $\begin{array}{c}1^{\circ} \\
\operatorname{PRE}(a)\end{array}$ \\
\hline Cicer arietinum L. & Goj & & $1^{\circ} ?$ \\
\hline Lens culinaris Medik. & $\begin{array}{l}\text { Kaíkjen (kajk'en) } \\
\text { Lenteja }\end{array}$ & Según FG significa "trozo pan o carne" & $\begin{array}{c}\text { DE } \\
\text { PRE (a) }\end{array}$ \\
\hline Medicago Iupulina L. & $\begin{array}{c}\text { Táshel jat } \\
\text { (t'ašel xa:t) }\end{array}$ & "Comida de vaca" (MC, dA, M) & $\mathrm{DE}$ \\
\hline $\begin{array}{l}\text { Phaseolus coccineus L. } \\
\text { (Ph. multiflorus var. albus) }\end{array}$ & $\begin{array}{c}\text { Ompójente, } \\
\text { omtejente (om } \\
\text { poxente, t'e:Xente) }\end{array}$ & $\begin{array}{l}\text { Provendría de "t'e:Xente" (flatulencia) y } \\
\text { de "poxenk" (oloroso) (FG). Aludiría a las } \\
\text { consecuencias de ingerir estos porotos }\end{array}$ & $\mathrm{DE}$ \\
\hline Phaseolus sp. & Awásh & $\begin{array}{l}(\mathrm{MC}, \mathrm{D}) \text {. Sería una } \\
\text { refonologización de "haba" }\end{array}$ & PRE \\
\hline Pisum sativum $\mathrm{L}$. & $\begin{array}{l}\text { Arveja } \\
\text { Verdétenk }\end{array}$ & "Verde" (MC) & $\begin{array}{l}\text { PRE (a) } \\
\text { DE }\end{array}$ \\
\hline $\begin{array}{c}\text { Prosopis cf. strombulifera Benth. } \\
\text { (Prosopis sp.) }\end{array}$ & Chẽn (č'en) & $\begin{array}{l}\text { "Mano" (dA, M, D, FG). Según D podría } \\
\text { aludir a la forma digitada de sus frutos }\end{array}$ & DE \\
\hline Vicia faba $\mathrm{L}$. & Awásh & $\begin{array}{l}(\mathrm{MC}, \mathrm{D}) \text {. Sería una } \\
\text { refonologización de "haba" }\end{array}$ & PRE \\
\hline $\begin{array}{c}\text { Vicia magellanica Hook. F. } \\
\text { (Vicia gramínea Sm., P. 121) (CTES) }\end{array}$ & $\begin{array}{l}\text { Óiwu jat } \\
\text { ('ojo xa:t) }\end{array}$ & $\begin{array}{l}\text { "Comida del ñandú" (Rhea } \\
\text { pennata) (MC, dA, M, D, FG) }\end{array}$ & DE \\
\hline \multicolumn{4}{|l|}{ GERANIACEAE } \\
\hline $\begin{array}{l}\text { Erodium cicutarium (L.) } \\
\text { L'Hér. ex Aiton }\end{array}$ & $\begin{array}{l}\text { Alfilerillo } \\
\text { guels }\end{array}$ & & $\begin{array}{l}\operatorname{PRE}(a) \\
1^{\circ}\end{array}$ \\
\hline \multicolumn{4}{|l|}{ GROSSULARIACEAE } \\
\hline Ribes magellanicum Poir. & $\begin{array}{l}\text { Kó'orr (k'o:'r) } \\
\text { Zarzaparrilla }\end{array}$ & "Pasto" (MC, dA, M, FG) & $\begin{array}{c}1^{\circ}(\mathrm{FV}) \\
\operatorname{PRE}(\mathrm{a})\end{array}$ \\
\hline
\end{tabular}




\section{G. F. Scarpa et al.- La etnobotánica Aonik’enk inédita de Raúl Martínez Crovetto}

Continuación Tabla 1

\begin{tabular}{|c|c|c|c|}
\hline $\begin{array}{l}\text { Especie actual (Identificación } \\
\text { de Martínez Crovetto) }\end{array}$ & $\begin{array}{l}\text { Fitónimo Aonik'enk } \\
\text { (notación IPA) }\end{array}$ & Significado y fuentes consultadas & $\begin{array}{l}\text { Tipo de } \\
\text { fitónimo }\end{array}$ \\
\hline \multicolumn{4}{|l|}{ IRIDACEAE } \\
\hline \multirow{3}{*}{$\begin{array}{l}\text { Olsynium junceum (E. } \\
\text { Mey. ex C. Presl) Goldblatt } \\
\text { (Sisyrrinchium sp., P. 88) }\end{array}$} & $\begin{array}{l}\text { Flor kóchenko } \\
\text { (flor koč'enk') }\end{array}$ & "Flor de la pampa lejana" (MC, FG) & $\mathrm{DE}$ \\
\hline & Kel jat (kel xa:t) & "Comida de la martineta" (MC, M, FG) & $\mathrm{DE}$ \\
\hline & $\begin{array}{l}\text { Élue jat } \\
\text { ('elwe xa:t) }\end{array}$ & $\begin{array}{c}\text { "Comida del pichón de avestruz" } \\
(\mathrm{MC}, \mathrm{dA}, \mathrm{M}, \mathrm{FG})\end{array}$ & DE \\
\hline \multicolumn{4}{|l|}{ JUNCACEAE } \\
\hline Juncus aff. balticus Willd. & Chéep (če:p) & $(\mathrm{MC}, \mathrm{FG})$ & $1^{\circ}$ \\
\hline (Juncus sp., P. 54, P. 106) (CTES) & ôt(e) ('ot) & $\begin{array}{l}\text { Según MC se trataría del nombre } \\
\text { propio de un ave paseriforme }\end{array}$ & $1^{\circ}$ \\
\hline Juncus sp. & K'or (k'or) & & $1^{\circ}(\mathrm{FV})$ \\
\hline \multicolumn{4}{|l|}{ LAMIACEAE } \\
\hline Mentha sp. & $\begin{array}{l}\text { Galal léen } \\
\text { (ga:lal le'n) }\end{array}$ & $\begin{array}{l}\text { "Lengua de vaca para tomar" (MC), } \\
\text { ya que "Galal" (planta de hojas } \\
\text { comestibles). y "Ie": agua, beber (dA, } \\
\text { D, M). Aludiría al consumo medicinal } \\
\text { de esta planta en forma de infusión }\end{array}$ & $2^{\circ}$ \\
\hline Prunella vulgaris $\mathrm{L}$. & $\begin{array}{l}\text { Yaláu } \\
\text { (šalaw) }\end{array}$ & $\begin{array}{l}\text { (MC, dA, M). Según FG su } \\
\text { nombre sería č’a:wt'. }\end{array}$ & $1^{\circ}$ \\
\hline \multicolumn{4}{|l|}{ NOTHOFAGACEAE } \\
\hline Nothofagus betuloides (Mirb.) Oerst. & $\begin{array}{l}\text { Kárro } \\
\text { (k'aro') }\end{array}$ & "Árbol”, “leño" (MC, M, D, FG) & $1^{\circ}(\mathrm{FV})$ \\
\hline \multicolumn{4}{|l|}{ PLANTAGINACEAE } \\
\hline Plantago sp.(P. 8; P. 33) (CTES) & $\begin{array}{l}\text { Kôřr (k'o'r) } \\
\text { Ôt(e), ('ot) }\end{array}$ & $\begin{array}{c}\text { "Pasto" } \\
\text { Según MC se trataría del nombre } \\
\text { propio de un ave paseriforme }\end{array}$ & $\begin{array}{l}1^{\circ}(\mathrm{FV}) \\
1^{\circ}\end{array}$ \\
\hline Plantago spp. (P. 91, P. 126) & Tímten jat & "Comida del avutarda" (MC, M) & DE \\
\hline \multicolumn{4}{|l|}{ PLUMBAGINACEAE } \\
\hline $\begin{array}{l}\text { Armeria maritima (Mill.) } \\
\text { Willd. (Armeria chilensis) }\end{array}$ & $\begin{array}{l}\text { Élue jat } \\
\text { ('elwe xa:t) }\end{array}$ & $\begin{array}{l}\text { "Comida del pichón de avestruz" } \\
\text { (MC, dA, M, D, FG) }\end{array}$ & $\mathrm{DE}$ \\
\hline $\begin{array}{l}\text { Limonium brasiliense (Boiss.) } \\
\text { Kuntze (Limonium sp., P. 32) (CTES) }\end{array}$ & $\begin{array}{l}\text { Guáikoro } \\
\text { Ómchel ('omč’el) }\end{array}$ & $\begin{array}{l}\text { Según MC sería una corrupción del término } \\
\text { "guaycurú", nombre criollo de dicha especie } \\
\text { (MC) }\end{array}$ & $\begin{array}{l}\text { PRE } \\
(\text { abs) } \\
1^{\circ}\end{array}$ \\
\hline \multicolumn{4}{|l|}{ POACEAE } \\
\hline $\begin{array}{c}\text { Agrostis stolonifera L. var. stolonifera } \\
\text { (Agrostis sp., P. 125) (SI) }\end{array}$ & $\begin{array}{l}\text { Kôr̆ } \\
\left(\mathrm{k}^{\prime} \mathrm{o}^{\prime} \mathrm{r}\right)\end{array}$ & "Pasto" & $1^{\circ}(\mathrm{FV})$ \\
\hline $\begin{array}{l}\text { Alopecurus magellanicus } \\
\text { Lam. var. magellanicus } \\
\text { (Alopecurus antarcticus) }\end{array}$ & $\begin{array}{c}\text { Shal(e) } \\
\text { (šalGe / "šalq") }\end{array}$ & $\begin{array}{l}\text { Provendría de "šalGe" / "šalq": manta, } \\
\text { mantillo (según FG), en alusión a } \\
\text { la alta cobertura de sus matas }\end{array}$ & $\mathrm{DE}$ \\
\hline Avena byzantina C. Koch & $\begin{array}{l}\text { Ot ko } \\
\text { ('ot k'o) }\end{array}$ & $\begin{array}{l}\text { "Flor de ôt" (MC). Provendría de "ôt(e)" } \\
\text { (nombre propio de Plantago sp., } \\
\text { Taraxacum sp. y Juncus aff. balticus) } \\
\text { y de "kot'am" (florecer) según FG. }\end{array}$ & $2^{\circ}$ \\
\hline $\begin{array}{l}\text { Bromus catharticus Vahl var. } \\
\text { catharticus (Bromus unioloides) } \\
\text { Bromus secalinus L. } 100\end{array}$ & $\underset{\text { Kôr̆ }}{\text { (k'o'r) }}$ & "Pasto" & $1^{\circ}(\mathrm{FV})$ \\
\hline Chusquea culeou E. Desv. & Séuru & $(\mathrm{MC}, \mathrm{M})$ & $1^{\circ}$ \\
\hline $\begin{array}{l}\text { Cortaderia araucana Stapf } \\
\text { Cortaderia selloana (Schult. \& } \\
\text { Schult. f.) Asch. \& Graebn. }\end{array}$ & $\begin{array}{l}\text { Télsen (telsen) } \\
\text { Telshm (telšm) }\end{array}$ & $(M C, d A, d G, M)$ & $1^{\circ}$ \\
\hline $\begin{array}{l}\text { Dactylis glomerata L. } \\
\text { Deschampsia sp. (Aira sp., P. 87) } \\
\text { (CTES) (Deschampsia sp., P. 86) }\end{array}$ & Kôřr (k'o'r) & "Pasto" & $1^{\circ}(\mathrm{FV})$ \\
\hline
\end{tabular}


Continuación Tabla 1

\begin{tabular}{|c|c|c|c|}
\hline $\begin{array}{l}\text { Especie actual (Identificación } \\
\text { de Martínez Crovetto) }\end{array}$ & $\begin{array}{l}\text { Fitónimo Aonik'enk } \\
\text { (notación IPA) }\end{array}$ & Significado y fuentes consultadas & $\begin{array}{l}\text { Tipo de } \\
\text { fitónimo }\end{array}$ \\
\hline $\begin{array}{c}\text { Elymus magellanicus } \\
\text { (Desv.) Á. Löve. } \\
\text { (Agropyron patagonicum, P. 75) } \\
\text { Elymus sp. } \\
\text { (Agropyron spp., P. 94, P. 96, P. 100) } \\
\text { Festuca acanthophylla E. Desv. } \\
\text { (Festuca sp., P. 80) (CTES) } \\
\text { Hordeum lechleri (Steudel) Schenk } \\
\text { (Hordeum sp., P. 41, P. 69) (CTES) } \\
\text { Hordeum pubiflorum (Hordeum } \\
\text { sp., P. 25, P. 30) }\end{array}$ & $\begin{array}{c}\text { Shal(e) } \\
\text { (šalGe / "šalq") }\end{array}$ & $\begin{array}{l}\text { Provendría de "šalGe" / "šalq": manta, } \\
\text { mantillo (según FG), en alusión a } \\
\text { la alta cobertura de sus matas }\end{array}$ & DE \\
\hline $\begin{array}{l}\text { Festuca gracillima Hook. f. } \\
\text { Hordeum spp. (P. 25; P. 30) } \\
\text { Lolium multiflorum Lam }\end{array}$ & Kôřr (k'o'r) & "Pasto" & $1^{\circ}(\mathrm{FV})$ \\
\hline Hordeum spp. (P. 25; P. 30) & $\begin{array}{l}\text { Guels } \\
\text { Éskel }\end{array}$ & $(\mathrm{MC}, \mathrm{M}, \mathrm{D})$ & $\begin{array}{l}1^{\circ} \\
1^{\circ}\end{array}$ \\
\hline Oryza sativa L. & Aros & Refonologización de "arroz" & PRE (a) \\
\hline $\begin{array}{l}\text { Pappostipa ibarii (Phil.) Romasch. } \\
\text { (Stipa sp., P. 17) (CTES) }\end{array}$ & $\begin{array}{c}\text { Shal(e) } \\
\text { (šalGe / "šalq") }\end{array}$ & $\begin{array}{l}\text { Provendría de "šalGe" / "šalq": manta, } \\
\text { mantillo (según FG), en alusión a } \\
\text { la alta cobertura de sus matas }\end{array}$ & DE \\
\hline & $\begin{array}{l}\text { Guels } \\
\text { Kôřr (k'o'r) }\end{array}$ & "Pasto" & $\begin{array}{c}1^{\circ} \\
1^{\circ}(\mathrm{FV})\end{array}$ \\
\hline $\begin{array}{c}\text { Poa annua L. } \\
\text { Poa sp. (sin datos) (P. } \\
\text { 37; 95) (CTES) } \\
\text { Puccinellia magellanica } \\
\text { (Hook. f.) Parodi (P. 26) } \\
\text { Stipa ibari Phil. (Stipa sp., P. 17) }\end{array}$ & Kôor (k'o'r) & "Pasto" & $1^{\circ}(\mathrm{FV})$ \\
\hline $\begin{array}{l}\text { Poa sp. (P. 76) (CTES) } \\
\text { Stipa sp. (P. 70) (CTES) }\end{array}$ & $\begin{array}{c}\text { Shal (e) } \\
\text { (šalGe / "šalq") }\end{array}$ & $\begin{array}{l}\text { Provendría de "šalGe" / "šalq": manta, } \\
\text { mantillo (según FG), en alusión a } \\
\text { la alta cobertura de sus matas }\end{array}$ & DE \\
\hline Stipa sp. (P. 70) (CTES) & Guels & & $1^{\circ}$ \\
\hline Triticum aestivum L. & $\begin{array}{l}\text { Káchilo (kačelo) } \\
\text { Kéukenk (kewkenk) }\end{array}$ & $\begin{array}{c}(\mathrm{MC}, \mathrm{dA}, \mathrm{D}) \\
\text { ("nombre antiguo") }\end{array}$ & $\begin{array}{l}1^{\circ} \\
1^{\circ}\end{array}$ \\
\hline Zea mays L. & Pío jat (pio xa:t) & "Comida de la gallina" (MC, dA, FG) & $\mathrm{DE}$ \\
\hline POLYGALACEAE & & & \\
\hline Polygala sp. (P. 30) & $\begin{array}{l}\text { Élue jat } \\
\text { ('elwe xa:t) }\end{array}$ & $\begin{array}{c}\text { "Comida del pichón de avestruz" } \\
(M C, d A, M, D, F G)\end{array}$ & DE \\
\hline $\begin{array}{c}\text { Polygala sp. } \\
\text { (sin datos, P. 102) (CTES) }\end{array}$ & $\begin{array}{c}\text { Kel jat } \\
\text { (kel xa:t) }\end{array}$ & "Comida de la martineta" (MC, M) & DE \\
\hline POLYGONACEAE & & & \\
\hline Polygonum aviculare $\mathrm{L}$. & Kôř (k'o'r) & "Pasto" & $1^{\circ}(\mathrm{FV})$ \\
\hline $\begin{array}{c}\text { Rumex acetosella L. (P. 29) (CTES) } \\
\text { Rumex sp. (P. 97) (CTES) }\end{array}$ & $\begin{array}{c}\text { Galal } \\
\text { (ga:lal) } \\
\text { Kâpenk kôř } \\
\text { (q'a:penk k'o'r) }\end{array}$ & $\begin{array}{l}\text { Nombre genérico asignado a } \\
\text { plantas con hojas comestibles } \\
\text { "Pasto colorado" (MC, dA, M) }\end{array}$ & $\begin{array}{l}1^{\circ} \text { (gen) } \\
2^{\circ}\end{array}$ \\
\hline Rumex sp. & $\begin{array}{l}\text { Kashél ktal } \\
\text { (t'ašel t'al) }\end{array}$ & $\begin{array}{c}\text { "Lengua de vaca" (de "t'ašel": vaca, } \\
\text { y t'al: lengua) (MC, dA, M) }\end{array}$ & $\operatorname{PRE}(t)$ \\
\hline RANUNCULACEAE & & & \\
\hline $\begin{array}{l}\text { Anemone multifida Poir. (P. 99) } \\
\text { Ranunculus sp. (P. 5) (CTES) }\end{array}$ & $\begin{array}{l}\text { Séperr } \\
\text { (seper) }\end{array}$ & $\begin{array}{l}\text { "Abrojo", "botón" (dA, M) Aludiría a las } \\
\text { infrutescencias en cabezuelas de esta } \\
\text { planta y a su condición de adherencia. }\end{array}$ & $\mathrm{DE}$ \\
\hline
\end{tabular}




\section{G. F. Scarpa et al.- La etnobotánica Aonik’enk inédita de Raúl Martínez Crovetto}

Continuación Tabla 1

\begin{tabular}{|c|c|c|c|}
\hline $\begin{array}{l}\text { Especie actual (Identificación } \\
\text { de Martínez Crovetto) }\end{array}$ & $\begin{array}{l}\text { Fitónimo Aonik'enk } \\
\text { (notación IPA) }\end{array}$ & Significado y fuentes consultadas & $\begin{array}{l}\text { Tipo de } \\
\text { fitónimo }\end{array}$ \\
\hline \multicolumn{4}{|l|}{ ROSACEAE } \\
\hline $\begin{array}{c}\text { Acaena confertissima Bitter } \\
\text { Acaena integerrima } \\
\text { Gillies ex Hook. \& Arn. } \\
\text { Acaena magellanica (Lam.) Vahl } \\
\text { Acaena ovalifolia Ruiz \& Pav } \\
\text { Acaena pinnatífida Ruiz \& Pav. } \\
\text { Acaena platyacantha Speg. } \\
\text { Acaena poeppigiana Gay } \\
\text { Acaena sericea J. Jacq. }\end{array}$ & $\begin{array}{l}\text { Abrojo, abrojito } \\
\text { Táperr, táparr } \\
\text { (taper, tapar) }\end{array}$ & $\begin{array}{l}\text { Según FG sería "nudo", "muñeca", "tobillo". } \\
\text { Según dA, M refiere a una "mata de laguna" }\end{array}$ & $\begin{array}{l}\text { PRE (a) } \\
\text { DE }\end{array}$ \\
\hline $\begin{array}{c}\text { Acaena magellanica (Lam.) Vahl } \\
\text { Acaena ovalifolia Ruiz \& Pav } \\
\text { Acaena sericea J. Jacq. }\end{array}$ & $\begin{array}{l}\text { Le kápenk (le' } \\
\text { q’a:penk) } \\
\text { Séperr (seper) }\end{array}$ & $\begin{array}{l}\text { "Agua roja" (inferimos que hace alusión a la } \\
\text { preparación que se ingiere como medicinal } \\
\text { contra gastralgias) (MC, dA, M, D, FG) } \\
\text { "Abrojo", "botón" (dA, M) Aludiría a las } \\
\text { infrutescencias en cabezuelas de esta } \\
\text { planta y a su condición de adherencia. }\end{array}$ & $\begin{array}{l}\mathrm{DE} \\
\mathrm{DE}\end{array}$ \\
\hline $\begin{array}{l}\text { Acaena pinnatífida Ruiz \& Pav. } \\
\text { Acaena platyacantha Speg. }\end{array}$ & $\begin{array}{l}\text { Le kápenk } \\
\text { (le' q'a:penk) }\end{array}$ & $\begin{array}{l}\text { "Agua roja" (inferimos que hace alusión a la } \\
\text { preparación que se ingiere como medicinal } \\
\text { contra gastralgias) (MC, dA, M, D, FG) }\end{array}$ & DE \\
\hline $\begin{array}{l}\text { Geum involucratum Pers. } \\
\text { (Geum sp.) (P. 118, P. 129) }\end{array}$ & $\begin{array}{l}\text { Kaámpter jât(en) } \\
\text { (k'a'mter xa:ten) }\end{array}$ & "Comida de la lagartija" (MC, M, D, FG) & DE \\
\hline $\begin{array}{l}\text { Potentilla chiloensis (L.) Mabb. } \\
\text { (Fragaria chiloensis) }\end{array}$ & $\begin{array}{l}\text { Chat, Chaut } \\
\text { (čat, čawt) }\end{array}$ & $(\mathrm{MC}, \mathrm{dA}, \mathrm{M}, \mathrm{D})$ & $1^{\circ}$ \\
\hline \multicolumn{4}{|l|}{ SCHOEPFIACEAE } \\
\hline $\begin{array}{c}\text { Arjona patagonica Hombr. \& Jacq. ex } \\
\text { Decne. (Arjona sp., P. 132) (CTES) } \\
\text { Arjona tuberosa Cav. } \\
\text { (Arjona sp., P. 145) (CTES) }\end{array}$ & $\begin{array}{l}\text { Saj } \\
\text { (sax) }\end{array}$ & $\begin{array}{l}\text { Nombre genérico de plantas } \\
\text { con tubérculos comestibles }\end{array}$ & $1^{\circ}(\mathrm{FV})$ \\
\hline \multicolumn{4}{|l|}{ SOLANACEAE } \\
\hline $\begin{array}{l}\text { Jaborosa magellanica } \\
\text { (Griseb.) Dusén }\end{array}$ & $\begin{array}{l}\text { Pojsh } \\
\text { (poxšk') }\end{array}$ & "Mal olor" (MC, FG) & DE \\
\hline $\begin{array}{l}\text { Lycium ameghinoi Speg. } \\
\text { (sin datos, P. 141) (CTES) }\end{array}$ & $\begin{array}{l}\text { Ttáparr } \\
\text { (tapar) }\end{array}$ & $\begin{array}{l}\text { Según dA, M es "mata de laguna" Según } \\
\text { FG sería "nudo", "muñeca", "tobillo" }\end{array}$ & DE \\
\hline $\begin{array}{l}\text { Lycium repens Speg. } \\
\text { (sin datos, P. 74) (CTES) }\end{array}$ & $\begin{array}{l}\text { Óiwu jat } \\
\text { ('ojo xa:t) }\end{array}$ & $\begin{array}{l}\text { “Comida del ñandú" (Rhea } \\
\text { pennata) (MC, dA, M, D, FG) }\end{array}$ & DE \\
\hline $\begin{array}{l}\text { Lycium repens Speg. (Lycium } \\
\text { sp.) (P. 140, P. 147) (CTES) }\end{array}$ & $\begin{array}{l}\text { Kaámpter jât(en) } \\
\text { (k'a'mter xa:ten) }\end{array}$ & "Comida de la lagartija" (MC, M, D, FG) & DE \\
\hline Nicotiana sp. (P. 120) & $\begin{array}{l}\text { Élue jat } \\
\text { ('elwe xa:t) }\end{array}$ & $\begin{array}{l}\text { "Comida del pichón de avestruz" } \\
\text { (MC, dA, M, D, FG) }\end{array}$ & DE \\
\hline Nicotiana tabacum L. & $\begin{array}{l}\text { Yáuch(e) (jawč') } \\
\text { Golk (golk) } \\
\text { Sálwe (salwe) }\end{array}$ & $\begin{array}{l}\text { Tabaco picado fino para cigarrillos (MC) } \\
\text { Tabaco picado grueso para pipa (MC) } \\
\text { Tabaco en plancha para picar o mascar } \\
\text { (MC). Según FG significa "mojado" }\end{array}$ & $\begin{array}{l}1^{\circ} \\
1^{\circ} \\
D E\end{array}$ \\
\hline Solanum tuberosum L. & Áshpal (ašpal) & $(\mathrm{MC}, \mathrm{dA}, \mathrm{D})$ & $1^{\circ}$ \\
\hline \multicolumn{4}{|l|}{ TROPAEOLACEAE } \\
\hline Tropaeolum patagonicum Speg. & $\begin{array}{l}\text { Chále } \\
\text { (čale) }\end{array}$ & $\begin{array}{c}\text { (MC, D). D indica que el fitónimo } \\
\text { "chajlü" se refiere Diposis patagonica } \\
\text { (Apiaceae), planta con la cual comparte } \\
\text { los tubérculos engrosados }\end{array}$ & $1^{\circ}$ \\
\hline \multicolumn{4}{|l|}{ URTICACEAE } \\
\hline $\begin{array}{l}\text { Urtica magallanica Poir. } \\
\text { Urtica sp. }\end{array}$ & Maken, Makon & & $1^{\circ}$ \\
\hline
\end{tabular}


Continuación Tabla 1

\begin{tabular}{|c|c|c|c|}
\hline $\begin{array}{l}\text { Especie actual (Identificación } \\
\text { de Martínez Crovetto) }\end{array}$ & $\begin{array}{l}\text { Fitónimo Aonik'enk } \\
\text { (notación IPA) }\end{array}$ & Significado y fuentes consultadas & $\begin{array}{l}\text { Tipo de } \\
\text { fitónimo }\end{array}$ \\
\hline \multicolumn{4}{|l|}{ VERBENACEAE } \\
\hline $\begin{array}{l}\text { Acantholippia seriphioides } \\
\text { (A. Gray) Moldenke } \\
\text { (sin datos, P. 72) }\end{array}$ & $\begin{array}{c}\text { Kapenk } \\
\text { (q'a:penk) } \\
\text { Té pampa } \\
\text { Keléen (k'ele:n) }\end{array}$ & $\begin{array}{l}\text { Sería una variante alofónica de "k'eler", } \\
\text { referida como planta medicinal según FG, } \\
\text { tal cual como es utilizada esta planta }\end{array}$ & $\begin{array}{c}\text { DE } \\
\text { PRE (a) } \\
1^{\circ}\end{array}$ \\
\hline $\begin{array}{l}\text { Junellia uniflora (Phil.) } \\
\text { Moldenke (sin datos, P. 21) }\end{array}$ & $\begin{array}{c}\text { Kel jat } \\
\text { (kel xa:t) }\end{array}$ & "Comida de la martineta" (MC, M) & DE \\
\hline $\begin{array}{c}\text { Junellia uniflora (Phil.) } \\
\text { Moldenke (Verbena uniflora, } \\
\text { Phacelia sp., P. 21) }\end{array}$ & $\begin{array}{l}\text { Terrsh } \\
\text { (terš) }\end{array}$ & $(\mathrm{MC}, \mathrm{D}, \mathrm{M}, \mathrm{dA})$ & $1^{\circ}$ \\
\hline $\begin{array}{l}\text { Mulguraea tridens (Lag.) } \\
\text { N. O'Leary \& P. Peralta } \\
\text { (Verbena tridens, P. 24) }\end{array}$ & $\begin{array}{l}\text { Kake, Sháke } \\
\text { (ša:k'e) Mata negra } \\
\text { Yénoi }\end{array}$ & “Leña” (MC, dA, M) & $\begin{array}{c}\text { DE } \\
\operatorname{PRE}(a) \\
1^{\circ}\end{array}$ \\
\hline Verbena sp. (P. 113) & Kálche (k’alče) & “Espinoso” (MC) & $1^{\circ}(\mathrm{FV})$ \\
\hline $\begin{array}{l}\text { Verbena sp. (sin datos, } \\
\text { P. 131) (CTES) }\end{array}$ & Ánkuis & & $1^{\circ}$ \\
\hline
\end{tabular}

Referencias. Fitonimia Aonik'enk, correspondencias botánicas y análisis léxico-nomenclatural. Abreviaturas: 10: Lexema primario; 20: Lexema secundario; CM: Diccionario de Casamiquela (2008); D: Néstor C. D.Huircapán (com. pers.); dA: Diccionario Aonik'enk (anónimo); DE: Nombre descriptivo o metafórico; dG: Diccionario Gününaküna (inédito); (FV): Forma de vida; FG: Diccionario de Fernández Garay (2004); (gen): Nombre genérico; M: Diccionario de Molina (1967); MC: Martínez Crovetto (inédito); PRE (a): Préstamo lingüístico absoluto; PRE (p): Préstamo lingüístico parcial; PRE (t): Préstamo lingüístico de traducción.

vasculares. El $88.5 \%$ de las mismas (146) son nativas o asilvestradas al área patagónica, mientras que sólo 19 resultaron exóticas cultivadas.

\section{Fitonimia Aonik'enk}

Como se puede apreciar en la Tabla 1, en varios casos hemos comprobado que vocablos referidos como fitónimos aludían en verdad al tipo de utilidad de la planta en cuestión. Tales es el caso de "jaie" (para Tropaeolum patagonicum Speg.) -sin traducción por parte de M.C.- pero que resultaría una variante fonética de "jatie" referido en los

Tabla 2. Cantidad de taxa de fanerógamas, líquenes y hongos por nivel de identificación.

\begin{tabular}{|cccc|}
\hline Taxa & Género & Especie & Taxa totales \\
\hline \# Taxa Fanerógamas & 32 & 123 & 155 \\
\# Taxa Lichenes & 1 & 3 & 4 \\
\# Taxa Fungi & 3 & 3 & 6 \\
Totales & $\mathbf{3 6}$ & $\mathbf{1 2 9}$ & $\mathbf{1 6 5}$ \\
\hline
\end{tabular}

diccionarios consultados como "comida" (Molina, 1967). Según Néstor Huircapán dicha denominación aludiría a la condición comestible de los tubérculos de esta especie (tal como ocurriría también con Diposis patagonica Skottsb. planta con la cual comparte estos órganos engrosados). El mismo criterio emplean para denominar a Mulguraea tridens (Lag.) N. O’Leary \& P. Peralta ("kake": literalmente "leña"). Confirman estas hipótesis los usos alimenticios y combustibles -respectivamentede estas dos especies, referidos en las anotaciones de M.C. Entre otros casos del mismo tipo se encuentran los fitónimos asignados a la "yerba" (Ilex paraguariensis A. St.-Hil.), uno de los cuales alude al recipiente prototípico que se utiliza para su consumo (o "mate" en lengua aonik'enk) y el otro a una preparación de la cual es objeto (denominada " $k$ 'omke(n)" a base de yerba y azúcar que se emplea como masticatorio). De la misma manera, tanto para aludir a Schinus marchandii F.A. Barkley como a Acantholippia seriphioides (A. Gray) Moldenke emplean los vocablos asignados a las bebidas con ellas elaboradas ("shâjen" y 


\section{G. F. Scarpa et al.- La etnobotánica Aonik'enk inédita de Raúl Martínez Crovetto}

"k'leen", respectivamente). Por lo anterior, todos estos vocablos no podrían ser considerados como verdaderos fitónimos, razón por la cual no han sido incorporados aquí como tales.

En otros casos el vocablo referido por M.C. como fitónimo refiere a la denominación de una de sus partes, tal como sucede con las especies de los géneros Schinus sp. y Arjona spp. En el primer caso fueron referidos los fitónimos "máke(n)", "mécharrn" y "mícharrn" que aluden a su resina utilizada como mascatorio, motivo por el cual fueron descartados como tales. En el caso de las especies de Arjona spp., en cambio, el vocablo referido ("saj") corresponde a un genérico con el que se nombran todas las plantas con tubérculos comestibles, razón por la cual lo consideramos aquí como fitónimo. En tres casos el fitónimo coincide con el nombre propio de un animal, como "kórpe" (Schoenoplectus californicus (C.A. Mey.) Soják y "zorrino macho"), “'ot" (Taraxacum sp. / Plantago sp. y un ave paserifome) y "k'aro" (Nothofagus betuloides (Mirb.) Oerst. y "carancho") (Molina, 1967; Fernández Garay, 2004). Las posibles explicaciones para esto último se discuten en el acápite correspondiente.

Finalmente, hemos detectado sobre el margen izquierdo de los cuadernos una lista de nombres criollos de varias plantas de la zona que M.C. habría anotado de su propio cuño a manera de ayuda mnemotécnica en sus encuestas con varios colaboradores. Esto es lo que inferimos los autores debido a que M.C. no refiere ningún fitónimo aonik'enk ni identificación botánica alguna sobre las mismas y, en la mayoría de los casos, ningún uso o significación específica (i.e. "ciprés", "maitén", "paico", "chaura", "lenga", "raulí" y "ñire", etc.). Los autores decidimos no incluir como fitónimos aonik'enk datos que M.C. registró de un informante nacido en Chiloé (Chile) proveniente del norte de la Patagonia (Sr. Galindo), debido a que ninguno de ellos fue consensuado por los colaboradores entrevistados, lo cual sería congruente con su procedencia foránea a la comunidad bajo estudio.

A partir de los criterios antes señalados pudimos identificar un total de 105 fitónimos en lengua aonik'enk, los cuales se enlistan en la segunda columna de la Tabla 1 tal cual como figura en la fuente primaria, seguido de la notación fonética aproximada según el International Phonetical Alphabet (IPA) entre paréntesis. Aunque en ocasiones se ha registrado más de un nombre vulgar para una misma entidad botánica, la situación inversa suele ser lo más frecuente, tal como lo sugiere la superioridad numérica de estas últimas respecto de los primeros (i.e. Acaena spp., Hordeum spp., Boopis spp., etc.).

\section{Correspondencias botánicas de los fitónimos Aonik'enk}

Las correspondencias botánicas de los fitónimos aonik'enk halladas en las fuentes primarias de este trabajo (fichas y libretas de campo de M.C.) fueron 173, contabilizando las identificaciones hasta género. Sin embargo, como resultado de los análisis antedichos -realizados tanto sobre las identificaciones botánicas como sobre la fitonimia-, los autores registramos 64 nuevas asociaciones entre la fitonimia aonik'enk y los nombres científicos de las plantas. En total se han evidenciado 236 correspondencias entre los taxa botánicos y la fitonimia aonik'enk, las cuales se enlistan en la Tabla 1 ordenadas por familia botánica.

\section{Clasificación fitonímica según categorías nomenclaturales}

En la tercera columna de la Tabla 1 se incluyen entre comillas las traducciones que en algunos casos indica M.C. sobre los fitónimos en lengua aonik'enk, además de una mención de los diccionarios consultados que refuerzan $-\mathrm{O}$ no- los significados de las mismas y, en ocasiones, un breve análisis semántico al respecto. En la cuarta columna de dicha tabla, en función del análisis anterior, se añade la categoría nomenclatural a la que pertenece cada uno de los fitónimos aonik'enk referidos, según los lineamientos establecidos en la metodología (lexemas primarios, secundarios, descriptivos y préstamos). En la Tabla 3 se cuantifican cada una de estas categorías, detallando para los lexemas primarios aquellos que representarían categorías etnoclasificatorias supragenéricas del tipo "forma de vida" tales como " $k$ 'álche" (espinosas), "sher" (matas), "k'o'r" (pasto), etc. Respecto a los préstamos identificados se distinguen entre aquellos que son absolutos, parciales o de traducción que han sido generalmente re-fonologizados respecto al castellano.

En los lexemas secundarios, los calificadores identificados (que acompañan a los lexemas primarios) frecuentemente remiten a alguna 
Tabla 3. Cuantificación de las categorias fitonímicas aonik'enk.

\begin{tabular}{|c|c|c|c|c|c|}
\hline & & & & \# fitónimos & \% fitónimos \\
\hline \multirow{7}{*}{$\begin{array}{l}\text { Clases de } \\
\text { fitónimos }\end{array}$} & \multirow{2}{*}{ Primarios } & Primario & 36 & \multirow{2}{*}{45} & \multirow{2}{*}{42.8} \\
\hline & & Forma de Vida & 9 & & \\
\hline & \multicolumn{3}{|l|}{ Secundarios } & 10 & 9.5 \\
\hline & \multicolumn{3}{|l|}{ Descriptivos } & 28 & 26.7 \\
\hline & \multirow{3}{*}{ Préstamos } & Parcial & 1 & \multirow{3}{*}{22} & \multirow{3}{*}{21.0} \\
\hline & & Absolutos & 17 & & \\
\hline & & Traducción & 4 & & \\
\hline \multicolumn{4}{|c|}{ Total } & 105 & 100 \\
\hline
\end{tabular}

característica organoléptica de la planta aludida tales como su color ("verdetenk": verde; " $k$ 'apenk": colorado, etc.), su intenso olor ("poxsh"), su tamaño (mata grande) o a un apéndice o parte de la misma (i.e. "barba de"; "raíz de", "lana de", etc.).

Respecto a los nombres descriptivos, las alusiones más utilizadas refieren a su condición de servir de alimento a un animal (i.e. óiwu jat: "comida del ñandú" = Senecio spp.) o a un "espíritu" determinado (i.e. ájchun jat: "comida del espíritu ájchun" = Agaricus sp.), o bien a alguna de sus características organolépticas tales como su color (i.e. $k$ 'âpenk $k$ 'ô'ř: "pasto colorado" = Rumex spp.), su intenso olor (i.e. am pojsh: "muy hediondo" = Artemisia magellanica Sch. Bip.) o su aspecto al tacto (i.e. wésen: "que corta" = Perezia recurvata (Vahl) Less. (Hook. \&Arn.) Cabrera). En menor medida, estos tipos de fitónimos hacen referencia a la morfología de sus frutos (i.e. séperr: "abrojo", "botón" = Boopis sp.), de sus flores (i.e. chóker: "bota" = Calceolaria sp.), a su forma de crecimiento (i.e. shal $(g)$ e: "cobertura" o "mantillo" = Alopecurus magellanicus Lam.) o a su condición utilitaria (i.e. sháke: "leña"= Mulguraea tridens). Tanto por la cantidad de las descripciones que incluyen como por el valor que estas representan, estos tipos de nombres aportan sustanciales datos para profundizar acerca de las significaciones de las plantas en base a tales indicios.

En la Tabla 3 se cuantifican cada una de las categorías y subcategorías fitonímicas aonik'enk identificadas y en la Fig. 3 se representan gráficamente los datos por categoría.

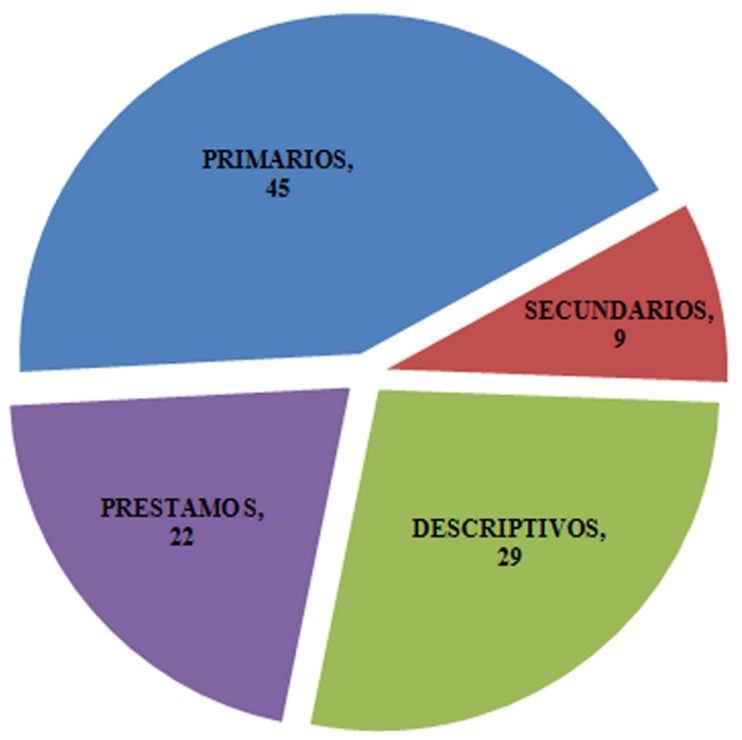

Fig. 3. Categorías nomenclaturales de los fitónimos aonik'enk.

\section{Discusión y Conclusiones}

A diferencia de otros sistemas nomenclaturales indígenas, la fitonimia aonik'enk evidencia un neto predominio de lexemas primarios (42.96 $\%$ ), seguido de los nombres descriptivos, de los préstamos y de los lexemas secundarios. La importancia de estos resultados radica en sus implicancias etnotaxonómicas, en cuanto a que los lexemas primarios (i.e. "algarrobo") suelen 


\section{G. F. Scarpa et al.- La etnobotánica Aonik'enk inédita de Raúl Martínez Crovetto}

cumplir el rol de (etno-) géneros y los secundarios el de etnoespecies (i.e. "algarrobo blanco") en los sistemas de clasificación vernáculos. La relación entre las dos últimas categorías en la fitonimia aonik'enk indica que la proporción de (etno-) géneros monotípicos $-33.4 \%$ - resulta similar a la registrada para otros grupos indígenas de Argentina -31\% para los chorotes-iyojwa'ja y 29 $\%$ para los chorotes-iyowujwa- (Scarpa, 2010).

Otras implicancias de los resultados obtenidos es que algunos de los nombres genéricos registrados por M.C. corresponden a verdaderas categorías etnoclasificatorias denominadas “formas de vida" -según la terminología etnotaxonómica empleada por Berlin (1992) para cientos de pueblos alrededor del mundo-, los cuales responden al criterio clasificatorio definido según atributos fisonómicos de la vegetación. Entre ellos figuran " $k$ 'álche" (espinosas), "sheř" (matas), “k’o'r”" (pasto), "cháun" (hongos arborícolas), "saj" (genérico de plantas con tubérculos comestibles), "áchame" (genérico de plantas bulbosas), "galal" (genérico asignado a plantas con hojas comestibles), " $m o$ " (genérico de plantas suculentas) y " $k$ 'arro" (genérico de plantas arbóreas o leñosas). La identificación de estas categorías posee una relevancia sustancial para ulteriores estudios acerca de la etnotaxonomía del pueblo aonik'enk.

La homologación identificada entre algunos fitónimos y zoónimos resulta un fenómeno frecuentemente registrado entre pueblos con religión de tipo animista. La razón de esta homologación estribaría en que según este sistema de creencias los aonik'enk conciben una consustanciación de tipo espiritual entre animales y plantas a nivel de la ontología propia de su cosmovisión.

Resulta significativo que solo Nothofagus betuloides sea el único árbol de los dominantes en el bosque subantártico que los aonik'enk asignen un fitónimo (k'arro), a pesar de la importancia manifiesta que estos poseen para los pobladores de dicha región. La justificación plausible que hallamos a este fenómeno, es que el espacio prototípico de los aonik'enk lo constituía la estepa patagónica -y su costa-, en detrimento de los bosques que ocupaban la porción occidental de su territorio. Esta explicación resulta congruente tanto con lo expresado por los gününa küna del Chubut como por las fuentes históricas consultadas (Martínič, 1995), en cuanto a que los tehuelches no se habrían adentrado tanto en los bosques occidentales.

Además de la importancia de los datos aquí referidos para el patrimonio cultural aonik'enk estos resultan especialmente valorables por el hecho de haber sido documentados hace ya más de 50 años, lo cual torna prácticamente imposible volver a registrarlos a campo en la actualidad debido al proceso de transformación sociocultural sufrido por este pueblo.

Se destaca, en conclusión, el gran volumen de nombres indígenas recabados (105) y de entidades botánicas a los que estos hacen referencia (165) en comparación con los antecedentes disponibles sobre la fitonimia del pueblo aonik'enk. De estos datos, el 28,4\% (47 taxa) corresponden a identificaciones botánicas obtenidas por los autores.

De la misma manera, resalta el gran número de correspondencias aquí referidos entre nombres aonik'enk y las entidades botánicas a las que estos hacen referencia (236), cada una de las cuales constituyen datos etnobotánicos propiamente dichos. En efecto, estas últimas significan un incremento en idéntica proporción de nuevas entradas léxicas para futuras recopilaciones del idioma aonik'enk. En función de todo lo señalado, se concluye que los datos presentados en este trabajo constituyen los registros etnobotánicos más exhaustivos hallados sobre los tehuelches de la provincia de Santa Cruz hasta la actualidad.

\section{Contribución de los Autores}

GFS halló el material inédito fuente de este artículo y consultó los ejemplares de herbario coleccionados por R. Martínez Crovetto en el IBONE de Corrientes. CNR y GFS transcribieron la información en la biblioteca del IBONE, ordenaron y sistematizaron la información en una base de datos y confeccionaron el mapa y la lámina de las Fig. 1 y 2 respectivamente. LMA y $\mathrm{NH}$ elicitaron los significados de los fitónimos en idioma aonik'enk. GFS, LMA y CNR clasificaron los fitónimos en categorías nomenclaturales, intepretaron los datos y escribieron el texto del artículo. 


\section{Agradecimientos}

A las autoridades del IBONE por las facilidades brindadas durante el registro de las fuentes primarias y la consulta de ejemplares de herbario aquí citados, especialmente a la Vicecuradora Carolina Peichoto y a los técnicos Walter Medina, Sandra y Débora Olmedo, así como a los encargados de su biblioteca. Al Consejo Nacional de Investigaciones Científicas y Técnicas y al Museo Argentino de Ciencias Naturales por facilitarnos sus instalaciones.

\section{Bibliografía}

ARENAS, P. 1992. Raúl Nereo Martínez Crovetto (19241988), su contribución a la etnobotánica y a los estudios americanistas. Suplemento Antropológico 27: $305-322$.

BERLIN, B. 1992. Ethnobiological classification. Principles of categorization of plants and animals in traditional societies. Princeton Univ. Press, Princeton. https://doi.org/10.1515/9781400862597.

CABI. 2019. CABI databases. [online]. Disponible en: http://www.speciesfungorum.org/ [Acceso: MayoJulio de 2019].

CASAMIQUELA, R. M. 1965. Rectificación $y$ ratificaciones. Hacia una interpretación definitiva del panorama etnológico de la Patagonia y área septentrional adyacente. Cuadernos del Sur, Univ. Nac. del Sur, Bahía Blanca.

CASAMIQUELA, R. M. 2008. Diccionario Tehuelche. Patagonia Sur Libros, Villa Adelina.

CENSABELLA, M. 1999. Las lenguas indigenas de la Argentina. Eudeba, Buenos Aires.

CORREA, M. N. 1984. Flora Patagónica. Tomo 8, Parte 4a. Dicotyledoneas dialipétalas (Salicaceae a Cruciferae). INTA, Buenos Aires.

DIARIO JORNADA. 2014. Chubut promueve la recuperación y revitalización de lenguas originarias. Edición del 13 Setiembre 2014. [online], Disponible en: https://www.diariojornada.com.ar/106383/ sociedad/chubut_promueve_la_recuperacion_y_ revitalizacion_de_lenguas_originarias/. [Acceso: 15 Agosto 2019].

DOMÍNGUEZ DÍAZ, E. 2010. Flora de interés etnobotánico usada por los pueblos originarios: Aónikenk, Selk'nam, Kawésqar, Yagan y Haush en la Patagonia Austral. Dominguezia 26: 19-29.
ELLEN, R. 2000. The cultural relations of classification. An analysis of Nuaulu animal categories from Central Seram. Cambridge Univ. Press, Cambridge. https://doi.org/10.1017/CBO9780511470530.

ESCALADA, F. 1949. El complejo "tehuelche". Estudios de etnografía patagónica. Coni, Buenos Aires.

FERNÁNDEZ GARAY, A. 1998. El tehuelche, una lengua en vías de extinción. Estudios Filológicos, Universidad Austral de Chile, Valdivia. https://doi.org/10.1086/466489.

FERNÁNDEZ GARAY, A. 2004. Diccionario Tehuelche-Español / Español-Tehuelche. Escuela de Investigación de Estudios Asiáticos, Africanos y Amerindios (CNWS), Univ. de Leiden, Leiden. https://doi.org/10.1075/s1.31.3.07hau

FLORA ARGENTINA. 2018. Base de datos "Flora Argentina". The Andrew W. Mellon Foundation, IBODA, IMBIV, INTA. [online]. Disponible en: http://www.floraargentina.edu.ar [Acceso: MayoJulio de 2019].

FRIEDBERG, C. 1991. Operative aspects of folk classification. In: PAWLEY, A. (ed.), Man and a half. Essays in Pacific Anthropology and Ethnobiology in honour of Ralph Bulmer, pp. 102109. The Polynesian Society, Auckland.

GBIF. 2019. GBIF.org. Global Biodiversity Information Facility. [online]. Disponible en: https://www.gbif. org/ [Acceso: Mayo-Julio de 2019].

HARRINGTON, T. 1946. Contribución al estudio del indio Gününa Küne. Revista Mus. La Plata, Secc. Antrop. 14: 237-275.

MARTINIČ, M. B. 1995. Los Aónikenk. Historia y Cultura. Universidad de Magallanes, Punta Arenas. Disponible en Memoria Chilena, Biblioteca Nacional de Chile http:/www.memoriachilena.gob. cl/602/w3-article-8404.html.

MOLINA, M. J. 1967. Antiguos pueblos patagónicos y pampeanos. Segunda parte. Léxico comparado. Anales Univ. Patagonia San Juan Bosco, Cs. Antrop. 1: 77-184.

MYCOBANK DATABASE. 2019. International Mycological Association. Fungal Databases, Nomenclature \& Species Banks. [online]. Disponible en: http://www.mycobank.org/ [Acceso: Mayo-Julio de 2019].

NACUZZI, L. R. 2005. Identidades impuestas. Tehuelches, aucas y pampas en el norte de la Patagonia. Sociedad Argentina de Antropología, Buenos Aires. 


\section{G. F. Scarpa et al.- La etnobotánica Aonik'enk inédita de Raúl Martínez Crovetto}

PIRONDO, A. \& H. A. KELlER. 2012. Raúl N. Martínez Crovetto: los albores de la etnobotánica en la Argentina. Introducción a Estudios Etnobotánicos 5. Bonplandia 21: 101-107. http://dx.doi.org/10.30972/bon.2121281

RAPOPORT, E. H., A. H. LADIO \& E. H. SANZ. 2001. Plantas nativas comestibles de la Patagonia Andina. Argentino-Chilena, Parte I. Universidad Nacional del Comahue, S.C. de Bariloche.

ROSSO, C. N. 2012. La etnobotánica de los grupos mocovíes de la reducción de San Javier, en el Gran Chaco, durante el siglo XVIII. Tesis Doctoral, Facultad de Filosofía y Letras, Univ. de Buenos Aires.

ROSSO, C. N. \& G. F. SCARPA. 2012. Identificaciones botánicas de las plantas empleadas entre los mocovíes en la reducción San Javier durante el siglo XVIII a partir de la obra de Florián Paucke, S. J. En: ARENAS, P. (ed.), Etnobotánica en zonas áridas y semiáridas del Cono Sur de Sudamérica, pp. 45-70. Sigma, Buenos Aires.

SCARPA, G. F. 2007. Hacia una etnotaxonomía vegetal Chorote I: Fitonimia, sistema nomenclatural y comparación dialectal. Suplemento Antropológico 42: 81-119.

SCARPA, G. F. 2010. Hacia una etnotaxonomía vegetal chorote II: Clasificación de las plantas entre las parcialidades iyojwa'ja y iyowujwa del Chaco argentino. En: MESSINEO, C.; G. F. SCARPA \& F. TOLA (comps.), Léxico y categorización etnobiológica en grupos indígenas del Gran Chaco, pp. 157-198. Univ. Nac. de La Pampa, Santa Rosa.

SCARPA, G. F. \& C. N. ROSSO. 2014. La etnobotánica moqoit inédita de Raúl Martínez Crovetto I: Descripción, actualización y análisis de la nomenclatura indígena. Bol. Soc. Argent. Bot. 49: 623-647. https://doi.org/10.30972/bon.232260
SECRETARÍA DE CULTURA DE LA NACIÓN. 2018. Los pueblos originarios en Argentina, hoy. [online]. Disponible en: https://www.cultura.gob.ar/ dia-internacional-de-los-pueblos-indigenas_6292/ [Acceso: 15 Agosto 2019].

SIFFREDI, A. \& M. MATARRESE. 2004. Espiritualidad tehuelche meridional (aónikenk). Recomponiendo las astillas de la memoria. En: CIPOLLETTI, M. S. (comp.), Los mundos de abajo. Los mundos de arriba. Individuo y sociedad en las tierras bajas, en los Andes y más allá, pp. 203-218. Abya-Yala, Quito.

SPEGAZZINI, C. 1884. Costumbres de los patagones. Conferencia dada en los salones de la Sociedad Científica Argentina el 2 de mayo de 1884. Anales Soc. Ci. Argent. 17: 221-240.

THE PLANT LIST. 2013. The Plant List. A working list of all plant species. Version 1.1. [online]. Disponible en: http://www.theplantlist.org/ [Acceso: Mayo de 2019].

TROPICOS.ORG. 2009. Tropicos. Missouri Botanical Garden. [online]. Disponible en: http://www. tropicos.org. [Acceso: Mayo de 2019].

VERA, J. 1991. Uso alimentario de recursos vegetales entre cazadores-recolectores de bahía Laredo, cabo Negro (Magallanes). Anales Inst. Patagonia, Ci. Soc. 20: 156-168.

VIEGAS BARROS, J. P. 1995. Zoonimia y etnozoología de los Pilagá, Toba, Mocoví, Mataco y Vilela. Raúl N. Martínez Crovetto. Instituto de Lingüística, U.B.A., Buenos Aires.

VIGNATI, M. A. 1936. Las culturas indígenas de Patagonia. En: LEVENE, R., Historia de la Nación Argentina I, pp. 591-645. Junta de Historia y Numismática Americana, Buenos Aires.

ZULOAGA, F. \& O. MORRONE. 2009. Flora del Cono Sur. Catálogo de las Plantas Vasculares. Instituto de Botánica "Darwinion", San Isidro. Disponible en: www2.darwin.edu.ar/Proyectos/FloraArgentina/ FA.asp. [Acceso: 15 de setiembre de 2019]. 
